\title{
Logarithmic Conformal Field Theory - OR - \\ How to Compute a Torus Amplitude on the Sphere
}

\author{
Micћael A.I. Flohr* \\ Physics Institute ${ }^{* *}$ \\ University of Bonn \\ Nussallee 12, D-53115 Bonn, Germany \\ flohr@th.physik.uni-bonn.de
}

July 14, 2021

\begin{abstract}
We review some aspects of logarithmic conformal field theories which might shed some light on the geometrical meaning of logarithmic operators. We consider an approach, put forward by V. Knizhnik, where computation of correlation functions on higher genus Riemann surfaces can be replaced by computations on the sphere under certain circumstances. We show that this proposal naturally leads to logarithmic conformal field theories, when the additional vertex operator insertions, which simulate the branch points of a ramified covering of the sphere, are viewed as dynamical objects in the theory.

We study the Seiberg-Witten solution of supersymmetric low energy effective field theory as an example where physically interesting quantities, the periods of a meromorphic one-form, can effectively computed within this conformal field theory setting. We comment on the relation between correlation functions computed on the plane, but with insertions of twist fields, and torus vacuum amplitudes.
\end{abstract}

In memoriam Ian Kogan.

\footnotetext{
${ }^{*}$ Work supported by the European Union network HPRN-CT-2002-00325 and in part by the string theory network (SPP no. 1096), Fl 259/2-2, of the Deutsche Forschungsgemeinschaft.

** On leave of absence from Institute for Theoretical Physics, Univesity of Hannover, Appelstr. 2, D-30167 Hannover, Germany.
} 


\section{Contents}

\begin{tabular}{lll}
\hline & Introduction & 3
\end{tabular}

\begin{tabular}{|lll}
2 & The approach of Knizhnik & 5
\end{tabular}

2.1 The Conformal Field Theory of $j$-Differentials . . . . . . . . . . . . . . . . . . . . . . 5

2.2 The Rational Logarithmic Conformal Field Theorv with $c=-2$. . . . . . . . . . . . 7

2.3 Logarithmic Operators . . . . . . . . . . . . . . . . . . . . . . . 12

2.4 Holomorphic Differentials . . . . . . . . . . . . . . . . . . . . . . . . . . . 15

3 Seiberg-Witten Solutions of Supersvmmetric Four-Dimensional Yang-Mills Theories 16

3.1 Periods of the Seiberg-Witten Differential . . . . . . . . . . . . . . . . . . . . . . . 18

3.2 Asvmptotics and $\mathrm{OPEs}$. . . . . . . . . . . . . . . . . . . . 20

4 The plane versus the torus $\quad 22$

4.1 Characters and torus amplitudes . . . . . . . . . . . . . . . . . . . . . . . 22

4.2 Periods and Torus Amplitudes . . . . . . . . . . . . . . . . . . . . . . . . . 23

4.3 Plane correlators and characters . . . . . . . . . . . . . . . . . . . . 26

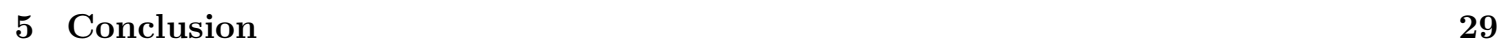

\begin{tabular}{|ll}
\hline A Structure Constants & 30
\end{tabular}

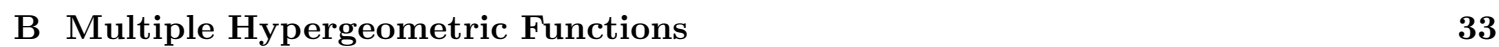




\section{Introduction}

Riemann surfaces belong to this special class of beautiful geometrical objects, whose members on one hand enjoy a sheer inexhaustible richness of mathematical structure, and one the other hand show up surprisingly (or even suspiciously?) often in the attempts of theoretical physicists to uncover fundamental patterns of what we call reality.

One of the more prominent appearances of Riemann surfaces in theoretical physics is, beyond any doubt, the worldsheet swept out by a string. Parameterization invariance in string theory then led us to conformal field theories (CFT) as the natural inhabitants of these worldsheets - and hence Riemann surfaces. A particular useful species of them, possessing a finite operator algebra, are the so-called rational conformal field theories (RCFTs), a concept first introduced with the minimal models of Belavin, Polyakov and Zamolodchikov [3] more than 15 years ago. Since then RCFTs established themselves as a main tool in modern theoretical physics, with deep relations to a multitude of mathematical fields.

String theories have changed much since their childhood, and their modern counterparts such as $M$-theory, unifying all the former theories, are now roamed by lots of other beings of higher dimensionality, branes and their worldvolumes. However, new developments in 4-dimensional effective field theories gave Riemann surfaces a new task. They now serve as the moduli spaces of the vacua of exactly solvable effective field theories, namely supersymmetric Yang-Mills theories. Strikingly, these effective field theories can be derived as certain low-energy limits of, e.g. type IIB string theory or $M$-theory. The fact that the moduli spaces turn out to be Riemann surfaces then is rooted in special properties of higher-dimensional geometrical objects such as Calabi-Yau compactifications in type IIB or the worldvolume of the 5-brane in $M$.

The main goal of this paper, which is devoted to the memory of Ian Kogan, is to test a more recently evolved species of CFTs for its suitability for theoretical physics - the so-called logarithmic conformal field theories (LCFTs). Although RCFTs nicely encode the additional degrees of freedom of string theories on their respective worldsheets, they completely fail to describe the physics in the moduli spaces of effective super-YM theories. This is simply due to the fact that RCFT correlation functions never ever produce logarithms, and hence cannot reproduce the logarithmic dependencies of the periods. Nonetheless, LCFTs do, and therefore we propose LCFTs as the natural candidates to encode the BPS spectrum of effective low-energy field theories.

The ideas we collect in this paper have partially been published in my earlier work [29]. Many discussions with Ian flow into that work. We both were very much intrigued by an old paper, written by Knizhnik. Knizhnik uses ghost system CFTs to compute string amplitudes up to two loops, i.e. genus two Riemann surfaces. The trick, Knizhnik uses, is to represent the complicated Riemann surfaces as a branched covering of the Riemann sphere and then simulate the effect of the branch points by special vertex operators. What made us so excited about Knizhnik's work is that he notes that certain correlators involving these branch point vertex operators may exhibit a logarithmic divergence. To my knowledge, this is the earliest mentioning of this possibility in the literature. Knizhnik discusses this issue only briefly, dismissing the possibility as, in his context correctly, physically irrelevant. Therefore, I find it appropriate to devote my paper to this common interest of both of us.

Logarithmic conformal field theories, first encountered and shown to be consistent in [37], are not just a peculiarity but merely a generalization of ordinary two-dimensional CFTs with broad and growing applications. One may well say that LCFTs contain ordinary rational conformal field theories as just the subset of theories free of logarithmic correlation functions. However, logarithmic divergences are sometimes quite physical, and so there is an increasing interest in these logarithmic conformal field theories.

The body of LCFT literature is by now too large to be listed here in completeness. We will here only note a few of the applications, confining ourselves to works which existed at the time when Ian and I discussed LCFT in connection with Seiberg-Witten theory. These are works on topics such as (multi-)critical polymers and percolation in two dimensions [15, 25, 41, 66, 73, twodimensional turbulence and magneto-hydrodynamics [27, 62, 72, the quantum Hall effect [26, 38, [57, 74, gravitational dressing [7, 49, 50, disorder and localization effects [12, 13, 53, 57, as well as 
the recoil problem of strings and $D$-branes [4, 17, 51, 52, 60] (see [59] and references therein for more recent work) and target-space symmetries in string theory in general [51.

There is also a growing body of literature on LCFT in general, where in particular all the powerful structures of RCFTs are either transfered or generalized to the logarithmic case. For example, LCFTs appear in the WZNW model on the supergroup $G L(1,1)$ [65], the $c_{p, 1}$ models (as well as non-minimal $c_{p, q}$ models) 25, 33, 37, 43, 64, gravitationally dressed conformal field theories [7], WZNW models at level 0 [51, 11] or fractional [34] or negative [54 level, and critical disordered models [12, 13, 39, 57. The references on the $c_{p, 1}$ models also contain considerable information on general questions such as characters, fusion rules, partition functions and null vectors (see also [28]), while logarithmic correlation functions were considered in general in [36, 49, 50, 58, 61, 71, see also 63 about consequences for Zamolodchikov's $C$-theorem. Generic construction schemes for LCFTs were discussed in 23 .

For more recent works and a more detailed exposition on the state of the art of LCFT, the reader is referred to the reviews [31, 35] and references therein.

The aim of this paper is to establish LCFTs as natural inhabitants of Riemann surfaces, providing a geometrical foundation for their special properties which distinguish them from ordinary CFTs. Furthermore, this is done with precisely such physical applications in mind, where Riemann surfaces play an important rôle, but ordinary RCFTs fail in effectively computing the desired information. Therefore, this paper will study LCFTs in the framework of certain classes of Riemann surfaces which, in particular, appear as the moduli spaces of vacua of exactly solvable supersymmetric Yang-Mills theories.

We will focus on the simplest case of non-trivial Riemann surfaces, the hyperelliptic ones. Such complex curves describe the moduli spaces of vacua of Seiberg-Witten models, i.e. exact solutions of low-energy $N=2$ supersymmetric Yang-Mills theories. Much of what is collected here can easily be generalized to the case of general Riemann surfaces with a global $\mathbb{Z}_{n}$ symmetry, which serve, for instance, as the moduli spaces of arbitrary four-dimensional supersymmetric gauge theories with reductive gauge groups, whose solutions have been found by Witten via $M$-theory.

The particular choice of our application, namely low-energy effective field theories, is motivated twofold: Firstly, these theories are of particular interest for the question of duality and for the formulation of high-energy theories (string theory, $M$-theory, etc.) which are phenomenological promising. Secondly, the so-called Seiberg-Witten models are good examples where one knows that a certain geometrical object, namely the hyperelliptic curve, encodes entirely all information about the theory, but where it can still be quite difficult to extract this information explicitly, meaning here to calculate the periods of certain forms.

Furthermore, this application illuminates the geometry behind logarithmic CFT. It is well known that vertex operators of worldsheet CFTs in string theory describe the equivalent of Feynman graphs with outer legs by simulating their effect on a Riemann surface as punctures. Now, in the new setting of moduli spaces of low-energy effective field theories, pairs of vertex operators describe the insertion of additional handles to a Riemann surface, simulating the resulting branch cuts. So, in much the same way as a smooth but infinitely long stretched tube attached to an otherwise closed worldsheet, standing for an external state, is replaced by a puncture with an appropriate vertex operator, so is a smooth additional handle, standing for an intersecting 4-brane on the 5-brane worldvolume (after switching on the 11-th dimension in $M$-theory), replaced by branch cuts with appropriate vertex operators at its endpoints.

The paper is structured as follows: Hence, section II reviews in general, how vertex operators can be used to describe the behavior of a $n$-ramified branch cut before concentrating on the hyperelliptic case and identifying the correct CFTs for the description of $j$-differentials. It is shown that in the case of our focus, this is the rational LCFT with $c=c_{2,1}=-2$. Finally, arbitrary Abelian differential 1-forms on hyperelliptic curves are defined in terms of conformal blocks of suitable vertex operators.

Section III gives a brief exposition of the Seiberg-Witten solutions of $N=2$ supersymmetric four-dimensional Yang-Mills theories before going on with the calculation of the periods of the meromorphic Seiberg-Witten differential. The case of gauge group $S U(2)$ is performed in some detail to show the naturality of the CFT approach and its potential to considerably simplify such 
computations.

The IV. section will then concentrate on the case of the torus in order to make the relation of Knizhnik's approach to objects, which one can compute directly on the torus, very explicit. For example, we will show there, that torus vacuum amplitudes can be evaluated directly and also as four-point functions on the sphere yielding exactly the same result. We also comment on the possibility to generalize this approach to other conformal field theories.

After the obligatory conclusions, an appendix presents all important structure constants of the $c=-2$ theory, which are needed when $n$-point correlation functions are expressed in terms of 4-point functions, as well as some information on multiple hypergeometric functions.

\section{The approach of Knizhnik}

The idea to compute integrals of differential forms on Riemann surfaces with the help of conformal field theory is actually not new. Some path-breaking papers on this idea are 6, 14, 48, 75]. However, as has been shown [55], the conformal field theories of twist and spin fields used in these earlier works are indeed very special logarithmic conformal field theories. As a consequence of this, we are now - after the advent of logarithmic conformal field theory - in a better position: Logarithmic conformal field theories possess a structure very close to rationality [25, 33] which allows us to make use of all the powerful tools available in rational conformal field theory. Not only can explicit calculations of period integrals be performed within the setting of degenerate conformal field theories à la BPZ [3], but also the physical interpretation of such periods, as in the Seiberg-Witten models, becomes more transparent. For example, the fusion rules yield a very simple and imaginative way to find the points in the moduli space of a Seiberg-Witten model, where certain states become massless. Moreover, the description within the logarithmic conformal field theory setting contains some surprising new structures not apparent in the older approach, especially the feature of Jordan-cell highest weight representations and the fact that the zero mode $L_{0}$ of the stress-energy tensor cannot be diagonalized.

The basic idea of the work of Knizhnik is that a given compact Riemann surface can always be represented as a $n$-fold ramified covering of the complex plane or, more precisely, its compactified version, the Riemann sphere $\mathbb{C P}^{1}$, for some number $n$. Thus, instead of computing a correlation function on the non-trivial Riemann surface, one could attempt to compute a correlator on the complex sphere with additional insertions of suitable operators which precisely have the effect of the branch points. Knizhnik explicitly constructed such vertex operators for the special case of the so-called ghost systems. These are particular conformal field theories of a free pair of anticommuting fields $b$ and $c$ with conformal scaling dimension $j$ and $1-j$ respectively. We briefly review his results here.

\subsection{The Conformal Field Theory of $j$-Differentials}

An arbitrary Riemann surface $X$ can be represented as a branched covering of $\mathbb{C P}^{1}$ where the covering map is denoted by $Z$. The metric on $X$ can be chosen as $g_{z z}=g_{\bar{z} \bar{z}}=0, g_{z \bar{z}}=1$. Under this choice, it was shown in [48 that each branch point $e_{i}$ corresponds to a particular primary field $\Phi_{i}\left(e_{i}\right)$. Usually, we will work with Riemann surfaces where the ramification numbers of all branch points are equal, say $n$, which means that $X$ has a global $\mathbb{Z}_{n}$ symmetry and can be defined by an equation

$$
y^{n}=\prod_{k=1}^{n m}\left(Z-e_{k}\right), \quad g=(n-1)\left(\frac{n m}{2}-1\right),
$$

in $\mathbb{C}^{2}=(y, Z)$. The hyperelliptic case, which will be the main scope of the present paper, corresponds to $n=2$, i.e. $\mathbb{Z}_{2}$ symmetry.

We now briefly review the construction of $j$-differentials and branch points in terms of primary fields. Near a branch point $a$ of order $n$, the covering map $Z$ takes locally the form $Z(y)=a+y^{n}$ yielding $n$ sheets of $X$ via the inverse map $y(Z)=(Z-a)^{1 / n}$, which we enumerate by $\ell=0, \ldots, n-1$. Thus, moving a point around $Z=a$, its inverse image moves $\ell \mapsto \ell+1 \bmod n$. We denote 
this analytic continuation operation by $\hat{\pi}_{a}$. For each sheet, we consider a pair $\phi^{(j), \ell}, \phi^{(1-j), \ell}$ of anticommuting fields of spin $j$ and $1-j$ respectively, and the action

$$
S^{(\ell)}=\int \phi^{(j), \ell} \bar{\partial} \phi^{(1-j), \ell} \mathrm{d}^{2} Z .
$$

In order that the anticommuting fields are local chiral fields, the spin $j$ must be (half-) integer. It is well known that the stress energy tensor takes the form

$$
T^{(\ell)}=-j \phi^{(j), \ell} \partial \phi^{(1-j), \ell}+(j-1) \phi^{(1-j), \ell} \partial \phi^{(j), \ell}
$$

giving rise to a central extension $c=c_{j} \equiv-2\left(6 j^{2}-6 j+1\right)$. The point is that under a conformal transformation $Z \mapsto Z^{\prime}(Z), \bar{Z} \mapsto \overline{Z^{\prime}(Z)}$, these fields transform as $j$ - and $(1-j)$-differentials respectively:

$$
\phi^{(j), \ell}\left(Z^{\prime}, \bar{Z}^{\prime}\right)\left(\frac{\mathrm{d} Z^{\prime}}{\mathrm{d} Z}\right)^{j}=\phi^{(j), \ell}(Z, \bar{Z})
$$

and analogously for $\phi^{(1-j), \ell}$. Here we assume that the operator product expansion (OPE) be normalized as

$$
\phi^{(j), \ell}\left(Z^{\prime}\right) \phi^{(1-j), \ell}(Z) \simeq \mathbb{I}\left(Z^{\prime}-Z\right)^{-1}+\text { regular terms }
$$

with $\mathbb{I}$ denoting the identity operator. Essentially, these fields are trivializing sections of $K^{j}$ on the Riemann surface $X$, where $K$ denotes the canonical line bundle. The central extension of the Virasoro algebra can than be expressed as $c=2 c_{1}\left[\operatorname{det} \bar{\partial}_{(j)}\right]$, i.e. by the first Chern class of the determinant of the holomorphic derivative acting on $j$-differentials.

The boundary conditions of these fields with respect to the operator $\hat{\pi}_{a}$ are for arbitrary $j \in \mathbb{Z} / 2$

$$
\hat{\pi}_{a} \phi^{(j), \ell}(Z)=(-)^{2 j} \phi^{(j), \ell+1 \bmod n}(Z) .
$$

In the vicinity of the branch point we can diagonalize $\hat{\pi}_{a}$ by choosing another basis for the $j$ differentials via a discrete Fourier transform,

$$
\phi_{k}^{(j)}=\sum_{\ell=0}^{n-1} \mathrm{e}^{-2 \pi \mathrm{i}(k+j-n j) \ell / n} \phi^{(j), \ell},
$$

such that $\hat{\pi}_{a} \phi_{k}^{(j)}=\mathrm{e}^{2 \pi \mathrm{i}(k+j-n j) / n} \phi_{k}^{(j)}$. As a consequence, we can define currents $J_{k}$ which are singlevalued functions of $Z$ in the vicinity of the branch point $a$. They are given by $J_{k}=: \phi_{k}^{(j)} \phi_{n-1-k}^{(1-j)}$ : and are chiral, $\bar{\partial} J_{k}=0$. These currents give rise to charges, and it turns out that a branch point carries the charges

$$
q_{k}=\frac{(n-1) j-k}{n}
$$

We may now perform a bosonization procedure expressing all fields with the help of $n$ analytic scalar fields $\varphi_{k}, k=0, \ldots, n-1$, normalized as $\left\langle\varphi_{k}(Z) \varphi_{l}\left(Z^{\prime}\right)\right\rangle=-\delta_{k l} \log \left(Z-Z^{\prime}\right)$. We find that, for instance, $\phi_{k}^{(j)}=: \mathrm{e}^{-i \varphi_{k}}:, \phi_{n-1-k}^{(1-j)}=: \mathrm{e}^{i \varphi_{k}}$ : and $J_{k}=\mathrm{i} \partial \phi_{k}$. One might attempt to diagonalize the stress-energy tensor too, obtaining

$$
T_{k}=-j \phi_{k}^{(j)} \partial \phi_{n-1-k}^{(1-j)}+(1-j) \phi_{n-1-k}^{(1-j)} \partial \phi_{k}^{(j)}=\frac{1}{2}\left(: J_{k} J_{k}:-(2 j-1) \partial J_{k}\right) .
$$

However, we will see later that under certain circumstances the zero mode of the stress energy tensor cannot be diagonalized. It follows that a branch point $a$ is represented by a vertex operator

$$
V_{\mathbf{q}}(a)=\exp (\mathbf{i q} \varphi(a)):, \quad \mathbf{q} \varphi=\sum_{k} q_{k} \varphi_{k}
$$

having conformal scaling dimension $h=h(\mathbf{q})=\sum_{k} h_{k}$ with the $h_{k}$ given by $h_{k}=\frac{1}{2}\left(q_{k}^{2}-(2 j-1) q_{k}\right)$. This corresponds to a Coulomb gas like construction of our conformal field theory (CFT) with a 
background charge $-2 \alpha_{0}$ given by $c_{j}=1-12 \alpha_{0}^{2}$, i.e. $2 \alpha_{0}=2 j-1$. The screening charges for the resulting degenerate model are $\alpha_{ \pm}=\alpha_{0} \pm \sqrt{\alpha_{0}^{2}+2}$. Actually, the full CFT consists of $n$ copies, one for each sheet, with total central charge $c=n c_{j}$.

In the case that $X$ has a global $\mathbb{Z}_{n}$ symmetry (1), all operators $\hat{\pi}_{e_{k}}$ can be diagonalized simultaneously, meaning that we can define our CFT globally, i.e. everywhere on the surface. Furthermore, the CFT is then just an $n$-fold tensor product of the simple CFT of two anticommuting analytic fields of spin $j$ and $(1-j)$ respectively. It is therefore sufficient to consider only one of these copies for most of our issues. Hence, we often will drop the indices $\ell$ or $k$. Clearly, according to (5), $\phi^{(1-j)}$ is the conjugate of $\phi^{(j)}$ with respect to the canonical scalar product $\langle\cdot, \cdot\rangle=\frac{1}{2 \pi \mathrm{i}} \oint \mathrm{d} z$. We also note that the OPE of the basic vertex operators has as its first term

$$
V_{\mathbf{q}}(a) V_{\mathbf{q}^{\prime}}\left(a^{\prime}\right) \simeq\left(a-a^{\prime}\right)^{\mathbf{q} \cdot \mathbf{q}^{\prime}} V_{\mathbf{q}+\mathbf{q}^{\prime}}\left(a^{\prime}\right)+\ldots .
$$

Usually, this is the leading term, since the order of the singularities on the right hand side is given by $h\left(\mathbf{q}^{\prime \prime}\right)-h(\mathbf{q})-h\left(\mathbf{q}^{\prime}\right)$ and has a minimum for $\mathbf{q}^{\prime \prime}=\mathbf{q}+\mathbf{q}^{\prime}$. Hence, correlation functions of vertex operators are simply evaluated as

$$
\left\langle\prod_{j=1}^{N} V_{\mathbf{q}_{j}}\left(z_{j}\right)\right\rangle=\prod_{i<j}\left(z_{i}-z_{j}\right)^{\mathbf{q}_{i} \cdot \mathbf{q}_{j}}
$$

with the charge balance condition $\sum_{i=1}^{N} \mathbf{q}_{i}=2 \alpha_{0} \mathbf{1}$.

What we have done is essentially defining a map from Riemann surfaces $X$ to the Fock space $\mathcal{F}$ of semi-infinite forms on the Hilbert space $\mathcal{H}=L^{2}\left(S^{1}\right)$. This map is called the string map. The Fock space is graded with respect to the charges $\mathbf{q}, \mathcal{F}=\bigoplus_{\mathbf{q}} \mathcal{F}_{\mathbf{q}}$, and admits a representation of the Virasoro algebra as the central extension of $\operatorname{Diff}\left(S^{1}\right)$. We have been a bit sloppy with the definition of vertex operators, since these are actually maps $V_{\mathbf{q}, \mathbf{p}}^{\mathbf{r}}(\cdot, Z): \mathcal{F}_{\mathbf{q}} \mapsto \operatorname{Hom}\left(\mathcal{F}_{\mathbf{p}}, \mathcal{F}_{\mathbf{r}}\right)$, called the screened chiral vertex operators. The screening refers to the fact that the 3-point function $\left\langle h_{\mathbf{r}}\left|Q_{-}^{\mathbf{s}_{-}} Q_{+}^{\mathbf{s}_{+}} V_{\mathbf{q}}(1)\right| h_{\mathbf{p}}\right\rangle$ is only non-zero, if $\mathbf{p}+\mathbf{q}+\mathbf{r}+\alpha_{-} \mathbf{s}_{-}+\alpha_{+} \mathbf{s}_{+}=\mathbf{0}$. Thus, $V_{\mathbf{q}, \mathbf{p}}^{\mathbf{r}}(\cdot, Z)$ denotes a vertex operator with the appropriate numbers of screening charges attached. The chiral primary fields are certain linear combinations of these chiral vertex operators with coefficients determined by locality and crossing symmetry of the primary fields of the complete CFT, combined from its left and right chiral half. In the following, we will denote by $\Phi_{\mathbf{q}}$ the primary fields, which are given as

$$
\Phi_{\mathbf{q}}(Z, \bar{Z})=\sum_{\mathbf{p}, \mathbf{r}} \mathcal{D}_{\mathbf{q}, \mathbf{p}}^{\mathbf{r}} V_{\mathbf{q}, \mathbf{p}}^{\mathbf{r}}(\cdot, Z) V_{\mathbf{\mathbf { q }}, \overline{\mathbf{p}}}^{\overline{\mathbf{r}}}(\cdot, \bar{Z}) .
$$

We will determine the coefficients $\mathcal{D}_{\mathbf{q}, \mathbf{p}}^{\mathbf{r}}$ for the case most important to us, $j=1$, in the Appendix A. These coefficients are closely related to the structure constants of the OPE, which can also be found in this Appendix. Let us further remark that, as evident from the construction, the CFT for the $j$-differentials is dual equivalent to the CFT for the $(1-j)$-differentials.

\subsection{The Rational Logarithmic Conformal Field Theory with $c=-2$}

In this paper, we concentrate on the $\mathbb{Z}_{2}$ symmetric case of Riemann surfaces, i.e. the case of hyperelliptic curves. Such a curve of genus $g$ can be represented by a double covering of the complex plane

$$
y^{2}=\prod_{k=1}^{2 g+2}\left(z-e_{k}\right), \quad \sum_{k=1}^{2 g+2} e_{k}=0,
$$

where we assume that $e_{k} \neq e_{l}$ for $k \neq l$ and that infinity is not a branch point. However, as we will see later, our formalism naturally incorporates the case of a degenerating curve (where two branch points flow together) as well as the case that infinity is a branch point. If we have made a choice for the branch cuts, we will display this by writing

$$
y^{2}=\prod_{k=1}^{g+1}\left(z-e_{k}^{-}\right)\left(z-e_{k}^{+}\right), \quad \sum_{k=1}^{g+1} e_{k}^{ \pm}=0,
$$


with the $g+1$ branch cuts running between $e_{k}^{-}$and $e_{k}^{+}$. Moreover, we mainly want to evaluate holomorphic or meromorphic 1-differentials, e.g. determine their periods. That means that we are interested in the case $j=1$. Then, the conformal field theory of twist fields appropriate for this case can be constructed out of two anticommuting fields of spin 1 and 0 , and has central charge $c=-2$. The branch points are represented by primary fields of conformal weight $h=-1 / 8$. This construction follows from first principles as shown in [48] and sketched in the preceding subsection. However, any conformal field theory with $c=-2$ and a primary field of conformal weight $h=-1 / 8$ is logarithmic 37.

Hence, we can associate a branch point with certain primary fields $\Phi_{q_{k}}$ having charges $q_{k}$ and conformal weights $h_{k}=\frac{1}{2}\left(q_{k}^{2}-q_{k}\right)$. It turns out that $\Phi_{q_{0}}=\Phi_{1 / 2}$ is a twist field, and $\Phi_{q_{1}}=\Phi_{0} \equiv \mathbb{I}$ is nothing else than the identity. Of course, the correct fields of the full conformal field theory for both sheets are the sums of the fields for each sheet, $\Phi_{\mathbf{q}}=\Phi_{\left(q_{0}, q_{1}\right)}$. Thus, the full conformal field theory has $c=2(-2)=-4$ with two identical versions for each field and is therefore a tensor product of two $c=-2$ theories. Since for the branch points $\mathbf{q}=(q, 0)$ is trivial on the second sheet, it follows that it is sufficient to consider only one copy of the $c=-2$ theories. $^{1}$

Let us now have a closer look at this logarithmic conformal field theory with $c=c_{2,1}=-2$, which is somehow the "first" model in the minimal series. Although the conformal grid is empty, one can consider the fields on its boundary. As was shown in 25, 33, there is a finite set of primary fields which is closed under the fusion rules. All $c_{p, 1}$ models, $p \in \mathbb{Z}_{+}, p>2$, are rational logarithmic conformal field theories whose conformal grid can formally be obtained by considering them as $c_{3 p, 3}$ models. In our case, we have five primary fields $\{\mathbb{I}, \mu, P, \sigma, J\}$ of conformal weights $h \in\left\{0,-\frac{1}{8}, 0, \frac{3}{8}, 1\right\}$ respectively. Note that there are two fields of the same conformal scaling dimension $h=0$. The field $\mathbb{I}$ is just the identity, while the field $P$ is closely related to the so-called puncture operator [49. The charges of these fields are then $\mathbf{q} \in\left\{(0,0),\left(\frac{1}{2}, 0\right),(1,0),\left(-\frac{1}{2}, 0\right),(-1,0)\right\}$ respectively, and the two screening currents carry charges $\alpha_{+}=2, \alpha_{-}=-1$. Note that the screening current $J_{-}$is identical to the primary field $J$, which is a special feature of logarithmic conformal field theory, where the screening charges become themselves local chiral primary fields [25]. The operator product expansion of any two fields $\Phi_{\mathbf{q}}, \Phi_{\mathbf{q}^{\prime}}$ with charges $\mathbf{q}=(q, 0), \mathbf{q}^{\prime}=\left(q^{\prime}, 0\right)$ has as its leading order

$$
\Phi_{\mathbf{q}}(z) \Phi_{\mathbf{q}^{\prime}}(w) \simeq(z-w)^{q q^{\prime}} C_{q, q^{\prime}}^{q+q^{\prime}} \Phi_{\mathbf{q}+\mathbf{q}^{\prime}}(w)+\ldots
$$

with $C_{q, q^{\prime}}^{q+q^{\prime}}$ denoting the structure constants of the operator algebra. We will freely use both notations of the primary fields, either $\Phi_{\mathbf{q}}(z)$ with $\mathbf{q}=(q, 0)$ the appropriate charge, or $\mathbb{I}(z), \mu(z)$ etc. The important thing to remember is that for $j=1$, the resulting CFT is a degenerate model. The subset of degenerate primary fields yields a closed operator algebra, and the corresponding charged Fock spaces admit well-defined screening charges. The admissible charges are given as

$$
q_{r, s}=\frac{1}{2}\left((1-r) \alpha_{+}+(1-s) \alpha_{-}\right)
$$

with $r, s$ non-negative integers. Since the $c=-2$ model is even rational, the conformal grid truncates, meaning $1 \leq r \leq 2,1 \leq s \leq 5$, which yields precisely the conformal weights

$$
h_{r, s}=\frac{1}{2}\left(q_{r, s}^{2}-q_{r, s}\right)
$$

given above. According to this formula, we may also express the OPEs (16) in terms of conformal weights instead of charges, namely

$$
\begin{aligned}
& \Phi_{r, s}(z) \Phi_{r^{\prime}, s^{\prime}}(w) \simeq \\
& (z-w)^{h_{r+r^{\prime}-1, s+s^{\prime}-1}-h_{r, s}-h_{r^{\prime}, s^{\prime}}} C_{h_{r, s}, h_{r^{\prime}, s^{\prime}}}^{h_{r+r^{\prime}-1, s+s^{\prime}-1}} \Phi_{r+r^{\prime}-1, s+s^{\prime}-1}(w)+\ldots
\end{aligned}
$$

We summarize all important data of the $c=-2$ rational LCFT in the following table, where $q^{*}$ denotes the charge of the conjugate field, $q^{*}=2 \alpha_{0}-q$, with respect to the standard (contravariant) pairing $\left\langle\Phi_{q} \mid \Phi_{p}\right\rangle$ of states $\left|\Phi_{p}\right\rangle \in \mathcal{F}_{p},\left\langle\Phi_{q}\right| \in \mathcal{F}_{q}^{*} \simeq \mathcal{F}_{q^{*}}$. By definition, $h\left(q^{*}\right)=h(q)$, and the

\footnotetext{
${ }^{1}$ This is a peculiarity of the $\mathbb{Z}_{2}$ case. The general case is more involved.
} 
background charge for $c=-2$ is $2 \alpha_{0}=1$. Keeping in mind that the fields $\sigma$ and $J$ are spin doublets results in a different choice for $q$ for them so that we have:

\begin{tabular}{|c||c|c|c|c|c|c|c|}
\hline$\Phi_{q}$ & $\mathbb{I}$ & $\mu$ & $P$ & $\sigma$ & $J$ & $J_{-}$ & $J_{+}$ \\
\hline \hline$(r, s)$ & $(1,1)=(2,5)$ & $(1,2)=(2,4)$ & $(1,3)=(2,3)$ & $(2,2)=(1,4)$ & $(2,1)=(1,5)$ & $(1,-1)=(2,7)$ & $(1,5)=(2,1)$ \\
\hline$h_{r, s}$ & 0 & $-1 / 8$ & 0 & $3 / 8$ & 1 & 1 & 1 \\
\hline$q_{r, s}$ & 0 & $1 / 2$ & 1 & $-1 / 2$ & -1 & -1 & 2 \\
\hline$q^{*}=q_{3-r, 6-s}$ & 1 & $1 / 2$ & 0 & $3 / 2$ & 2 & 2 & -1 \\
\hline$\alpha_{-} q$ & 0 & $-1 / 2$ & -1 & $1 / 2$ & 1 & 1 & -2 \\
\hline$\alpha_{-} q^{*}$ & -1 & $-1 / 2$ & 0 & $-3 / 2$ & -2 & -2 & 1 \\
\hline$\alpha_{+} q$ & 0 & 1 & 2 & -1 & -2 & -2 & 4 \\
\hline$\alpha_{+} q^{*}$ & 2 & 1 & 0 & 3 & 4 & 4 & -2 \\
\hline
\end{tabular}

Note that all fields are local with respect to the screening currents, and that all fields are at worst "half-local" with respect to any other field, which makes perfect sense on a Riemann surface which is just a branched double covering of the complex plane.

For a very thoroughly exposition of the $c=-2$ model see e.g. [38. The field $\mu$ is best viewed as carrying half a branch cut with it. Therefore, only correlation functions with an even number of these twist fields (or its excitation $\sigma$ ) will be non-zero, since these fields have to come in pairs creating the branch cuts. However, there is no direct way to tell which twist field joins with which. On the contrary, the conformal blocks are in one-to-one correspondence with a set of independent ways of distributing the branch cuts between the twist fields. This is illuminated by the fact that the operator product expansion of two twist fields is $[\mu] \star[\mu]=[\mathbb{I}]+[P]$. Indeed, suppose we join two twist fields with no branch cut between them, which just amounts in connecting two branch cuts to one, decreasing the genus of the surface by one. The point in the middle, where the two twist fields joined, can be pulled out of the new branch cut, and because this point does not have any special property anymore, it is best described by attaching the identity operator $\mathbb{I}$ to it. On the other hand, if two twist fields, which are connected by a branch cut, are joined, one branch cut shrinks to zero size, thus changing a genus $g$ surface to a genus $g-1$ surface with one puncture. Clearly, this is the case where the puncture operator $P$ comes into play. This operator is the logarithmic partner of the identity and creates the logarithmic divergences in correlation functions. In fact, we have the following important 2-point functions:

$$
\begin{aligned}
\langle\mathbb{I}(z) \mathbb{I}(w)\rangle & =0 \\
\langle\mathbb{I}(z) P(w)\rangle & =1 \\
\langle P(z) P(w)\rangle & =-2 \log (z-w) .
\end{aligned}
$$

The $c=-2$ theory of the $j=1$ differentials admits a free field realization with one scalar field $\varphi_{k}$ per sheet, $k=0,1$ in the hyperelliptic case. For later convenience, we now prepare a further computational tool. It is possible to use the same scalar fields to construct the $c=1$ conformal field theory of the $j=\frac{1}{2}$ differentials with fields $\Phi_{\tilde{\mathbf{q}}}(z)$ which carry half the charges of the $c=-2$ fields $\Phi_{\mathbf{q}}(z)$ do: If $\mathbf{q}=(q, 0)$, then $\tilde{\mathbf{q}}=\left(\frac{q}{2},-\frac{q}{2}\right)$. To this end, we first introduce the vertex operators for the branch points for the case that $j$ is half-integer, which now split into pairs

$$
\begin{aligned}
& V_{+}(a)=\exp \left(\mathrm{i} \frac{j}{2} \varphi_{0}+\mathrm{i} \frac{j-1}{2} \varphi_{1}\right), \\
& V_{-}(a)=\exp \left(\mathrm{i} \frac{j-1}{2} \varphi_{0}+\mathrm{i} \frac{j}{2} \varphi_{1}\right),
\end{aligned}
$$

and define $\Phi_{\tilde{\mathbf{q}}}=\Phi_{\left( \pm \frac{1}{4}, \mp \frac{1}{4}\right)}(a)$ as the corresponding primary fields for the case $j=\frac{1}{2}$. In a similar fashion, the other fields $\Phi_{\tilde{\mathbf{q}}}(z)$ can be constructed. ${ }^{2}$ Hence, we have $\tilde{\mathbb{I}}=\Phi_{(0,0)} \equiv \mathbb{I}, \tilde{\mu}=\Phi_{\left(\frac{1}{4},-\frac{1}{4}\right)}$,

\footnotetext{
${ }^{2}$ Of course, the charges of the conjugate fields are different, namely $q^{*}=-q$, for the $c=1$ theory, since the background
} 
$\tilde{P}=\Phi_{\left(\frac{1}{2},-\frac{1}{2}\right)}, \tilde{\sigma}=\Phi_{\left(-\frac{1}{4}, \frac{1}{4}\right)}$, and $\tilde{J}=\Phi_{\left(-\frac{1}{2}, \frac{1}{2}\right)}$, with the conformal weights $h \in\left\{0, \frac{1}{16}, \frac{1}{4}, \frac{1}{16}, \frac{1}{4}\right\}$ respectively. Clearly, an arbitrary correlation function of the free $c=1$ theory is simply

$$
\left\langle\prod_{j=1}^{N} \Phi_{\tilde{\mathbf{q}}_{j}}\left(z_{j}\right)\right\rangle_{c=1}=\prod_{i<j}\left(z_{i}-z_{j}\right)^{\frac{1}{2} q_{i} q_{j}}
$$

subject to the condition that $\sum_{j=1}^{N} q_{j}=0$ (otherwise, appropriate screening charges must be introduced, or, put in a different language, the fermionic zero modes must be absorbed by additional fields $\phi_{k}^{\left(j=\frac{1}{2}\right)}, \phi_{1-k}^{\left(1-j=\frac{1}{2}\right)}$ inserted into the correlator). We may further divide this $c=1$ theory out of our $c=-2$ theory with the effect that the free part of correlation functions is canceled,

$$
\left\langle\left\langle\prod_{j=1}^{N} \Phi_{q_{j}}\left(z_{j}\right)\right\rangle\right\rangle \equiv \frac{\left\langle\prod_{j=1}^{N} \Phi_{\left(q_{j}, 0\right)}\left(z_{j}\right)\right\rangle_{c=-2}}{\left(\left\langle\prod_{j=1}^{N} \Phi_{\left(\frac{1}{2} q_{j},-\frac{1}{2} q_{j}\right)}\left(z_{j}\right)\right\rangle_{c=1}\right)^{2}} .
$$

These reduced correlators will be the objects we are most interested in the following. The reason is that we will express period integrals over meromorphic differential forms in terms of correlation functions. However, a correlation function always consists of two parts, firstly the free contribution (sometimes also called the classical part), and secondly a non-trivial contribution (sometimes called the quantum part) involving all monodromy properties. This latter part can be written as certain contour integrals over the screening charges, if the CFT is a degenerate model, and it is this latter part which will provide us with the desired information. So, the above construction is a shorthand for throwing away what we do not need.

The careful reader will note that naively the charge balance for a $c=-2$ correlator should be $\sum_{j=1}^{N} q_{j}=2 \alpha_{0}=1$ (including all screening charges or zero-mode absorbing pairs of anti-commuting $j,(1-j)$ fields) due to the non-vanishing background charge. Indeed, as is demonstrated e.g. in 38, correlation functions of the $c=-2$ theory are non-zero only when one field $P$ is put at infinity, which nicely accounts for the background charge. The reason is that in the $c=-2$ theory we have the non-trivial vacuum structure $\langle\mathbb{I} \mid \mathbb{I}\rangle=0$, and $\langle P \mid \mathbb{I}\rangle=1$. However, due to the duality between $j$ and $(1-j)$, we could also impose the charge balance condition $\sum_{j=1}^{N} q_{j}=-2 \alpha_{0}=-1$, which simply amounts in replacing $\langle-1|$ by $\langle-1| Q_{+}=\langle 1|$. The validity of this is ensured, because the screening charge $Q_{+}$may act locally at infinity, and since $\langle-1|$ is a spin doublet state. This, together with the fact that, in particular, the branch point field $\mu$ is self-conjugate, leads to the following important fact: Any correlation function $\left\langle\prod_{j=1}^{N} \Phi_{\left(q_{j}, 0\right)}\left(z_{j}\right)\right\rangle_{c=-2}$ contains at least one conformal block involving precisely one more screening charge $Q_{-}$to ensure the correct charge balance, than its companion correlation function $\left\langle\prod_{j=1}^{N} \Phi_{\left(q_{j} / 2,-q_{j} / 2\right)}\left(z_{j}\right)\right\rangle_{c=1}$. As a consequence, the above expression (23) precisely extracts this one integration $Q_{-}=\oint J_{-}(z)$ over the additional screening current. ${ }^{3}$

As a matter of fact, whenever the charge balance of the primary fields is zero, the denominator of (23) takes the simple form (22). Otherwise, since we are only interested in such conformal blocks which involve precisely one screening integration, we will implicitly assume that the

charge $\alpha_{0}=0$ for $c=1$. The $c=1 \mathrm{CFT}$ with primary fields given by the $c=-2$ charges can be identified as the CFT of the Dirac fermion with compactification radius $R=1 / \sqrt{2}$. Since branch points have now two vertex operators associated with them, $\Phi_{\frac{1}{4}}$ and $\Phi_{-\frac{1}{4}}$, they allow for different boundary conditions. By saying that we choose the charges to be the same as for the $c=-2$ theory, we fix the boundary conditions to be the completely periodic ones.

${ }^{3}$ Note that equation (23) is related to a well known formula for the determinants of $\bar{\partial}_{(j)}$, namely $\operatorname{det}_{\mathbf{m}} \bar{\partial}_{(j=1 / 2)}\left(\operatorname{det} \bar{\partial}_{(j=0)}\right)^{1 / 2}=\theta_{\mathbf{m}}$, where the characteristics $\mathbf{m}=\left(\mathbf{m}^{\prime}, \mathbf{m}^{\prime \prime}\right)$ defines the boundary conditions imposed on the fermions, i.e. $j=\frac{1}{2}$-differentials, and where $\theta_{\mathbf{m}}$ is the corresponding theta-constant. An alternative but equivalent definition can be given, which entirely remains within the $c=-2$ realm and, moreover, has a direct physical interpretation in terms of partition functions. Since we want to perform integrals with just one screening charge, we may write $\left\langle\left\langle\prod_{j} \Phi_{q_{j}}\left(z_{j}\right)\right\rangle\right\rangle=\left.\frac{\partial}{\partial \beta} \log \left\langle\prod_{j} \Phi_{q_{j}}\left(z_{j}\right) \exp \left(\beta \int J_{-}(z) \hat{c}\right)\right\rangle\right|_{\beta=0}$. Here, $\hat{c}$ stands for the necessary cocycle which accounts for the shift in momentum. This latter definition could easily be generalized towards extracting several screening integrations. 
charge balances are ensured via insertion of appropriate numbers of zero mode absorbing fields, $\phi_{k}^{(j=1)}\left(Z_{m}\right), \phi_{1-k}^{(1-j=0)}\left(Z_{m}^{\prime}\right)$ in the $j=1$ numerator, and $\phi_{k}^{\left(j=\frac{1}{2}\right)}\left(Z_{n}\right), \phi_{1-k}^{\left(1-j=\frac{1}{2}\right)}\left(Z_{n}^{\prime}\right)$ in the $j=\frac{1}{2}$ denominator respectively, and taking a regularized limit $Z_{m}, Z_{m}^{\prime}, Z_{n}, Z_{n}^{\prime} \longrightarrow \infty$. We remark that the $c=-2$ theory of $j=1$ differentials can formally be written as a coset $\left(\widehat{S U(2)}_{-1} \times \widehat{\left.S U(2)_{1}\right) / \widehat{S U(2)}}\right.$, where the the $\widehat{S U(2)}$, stems from the $j=\frac{1}{2}$ contribution with $c=1$. It is therefore tempting to interpret equation (23) in the sense that this part can (formally) be factored out.

Last, but not least, we would like to note how the correlators $\langle\langle\cdot\rangle\rangle$ behave under a global conformal transformation of the coordinates. Let $M=\frac{a Z-b}{c Z-d}$ with $a d-b c \neq 0$ be a global conformal transformation (on the Riemann sphere). A generic CFT correlation function of primary fields transforms then as $\left\langle\prod_{j} \phi_{j}\left(z_{j}\right)\right\rangle=\prod_{i}\left(\left.\frac{\partial M(Z)}{\partial Z}\right|_{Z=z_{i}}\right)^{-h_{i}}\left\langle\prod_{j} \phi_{j}\left(M\left(z_{j}\right)\right)\right\rangle$. On the other hand, we have to keep in mind that we throw away the free part of the correlation functions. The only remaining contributions come from the integrations with the screening currents. This means that the total exponents are $-h_{i,(c=-2)}+2 h_{i,(c=1)}=-\left(q_{i}^{2}-2 \alpha_{0} q_{i}\right) / 2+2 q_{i}^{2} / 4=q_{i} / 2$. Therefore, the correlators defined in (23) transform under $M$ according to

$$
\left\langle\left\langle\prod_{j=1}^{N} \Phi_{q_{j}}\left(z_{j}\right)\right\rangle\right\rangle=\prod_{i=1}^{N}\left(\left.\frac{\partial M(Z)}{\partial Z}\right|_{Z=z_{i}}\right)^{\frac{q_{i}}{2}}\left\langle\left\langle\prod_{j=1}^{N} \Phi_{q_{j}}\left(M\left(z_{j}\right)\right)\right\rangle\right\rangle .
$$

If zero-mode absorbing spin $j$ fields, i.e. $j$-differentials, are inserted, these will contribute an additional factor - in the limit - of $\lim _{z \rightarrow \infty}\left[\left(\partial_{z} M(z)\right) \frac{z^{2}}{M(z)^{2}}\right]^{q(j) / 2}$ with $z$ the localization variable and $q(j)$ the charge of the $j$-differential at infinity. For example, to switch the charge balance condition from $\sum_{i} q_{i}=1$ to $\sum_{i} q_{i}=-1$, the zero modes are absorbed with the help of $J_{+} \sim: P^{2}$ : acting at infinity having charge $q\left(J_{+}\right)=2$. We will often omit these zero mode absorbing fields, since they can be inferred from the charge balance condition and the requirement that only one screening current integration is to be performed.

We will later express correlation functions in terms of generalized hypergeometric functions of several variables. As shown in Appendix B, these will depend on the inverse crossing ratios, i.e. on $M\left(z_{j}\right)^{-1}$. Assuming that $M$ maps $\left\{z_{1}, z_{2}, z_{3}\right\} \mapsto\{\infty, 1,0\}$, the generalized hypergeometric functions $F$ will depend explicitly only on $M\left(z_{4}\right)^{-1}, \ldots, M\left(z_{N}\right)^{-1}$. Therefore, the above formula applied to $F$ will be modified to

$$
\begin{aligned}
\left\langle\left\langle\prod_{j=1}^{N} \Phi_{q_{j}}\left(z_{j}\right)\right\rangle\right\rangle & =\prod_{i=1}^{3}\left(\partial_{z_{i}} M\left(z_{i}\right)\right)^{\frac{q_{i}}{2}} \prod_{i=4}^{N}\left(\frac{\partial_{z_{i}} M\left(z_{i}\right)}{M\left(z_{i}\right)^{2}}\right)^{\frac{q_{i}}{2}} \lim _{z \rightarrow \infty}\left(z^{2} \frac{\partial_{z} M(z)}{M(z)^{2}}\right)^{Q} \\
& \times F\left(M\left(z_{4}\right)^{-1}, \ldots, M\left(z_{N}\right)^{-1}, M(z \rightarrow \infty)^{-1}\right)
\end{aligned}
$$

including the contribution for the zero mode absorbing fields, and where we have put $Q=1-$ $\frac{1}{2} \sum_{i} q_{i}$. We will often use the notation $x(z) \equiv 1 / M(z)$ for the inverse crossing ratios.

Similar considerations hold for the power exponents in OPEs, when these are performed within a $\langle\cdot\rangle$ correlator. One finds

$$
\begin{aligned}
& \left\langle\ldots \Phi_{q_{1}}(z) \Phi_{q_{2}}(w) \ldots\right\rangle \sim \\
& \sum_{q \geq 0} C_{h(q), h\left(q_{2}\right)}^{h(q)}(z-w)^{\frac{1}{2}\left(q-q_{1}-q_{2}\right)\left(q-1+q_{1}+q_{2}\right)} \\
& \sum_{\{r\}}^{h(q) ;\{r\}}(z-w)^{1-h(q)_{\min }} \\
& \quad a_{h\left(q_{1}\right), h\left(q_{2}\right)}^{h(z\} \mid \geq-1-(\Delta h(q))} \\
& \quad \times\left(\partial^{-1-(\Delta h(q))-|\{r\}|}(z-w)^{h(q)_{\min }-1}\right)\left\langle\ldots \mathcal{L}_{-\{r\}} \Phi_{q}(w) \ldots\right\rangle .
\end{aligned}
$$

This form of the OPE also shows the descendant terms and does explicitly take into account possible logarithmic divergences due to different fields of the same conformal scaling dimension (or fields 
whose conformal dimensions differ by integers). This happens precisely when $q$ is a positive integer. We then have that $h(q)=h(q)_{\min }+(\Delta h(q))$ with $h(q)_{\text {min }}$ the conformal scaling dimension of the lowest primary field within a Jordan block. Negative powers of derivatives simply mean formal term by term integration of a generalized series expansion (which may contain fractional powers, logarithms, etc.), and $\mathcal{L}_{-\{r\}}$ is a shorthand for $L_{-r_{1}} L_{-r_{2}} \ldots L_{-r_{n}}$. A more rigorous formulation of the OPE of logarithmic CFTs can be found in 30. Linearity is ensured if the OPE is applied before the $\langle\cdot\rangle$ expectation values are taken.

\subsection{Logarithmic Operators}

The CFT of the spin $(1,0)$ ghost system with central charge $c=-2$ has the great advantage that the origin of logarithmic operators is well understood. It is the presence of the field $\mu$, which in our geometrical setting simulates branch points, which inevitably leads to the appearance of the logarithmic operator $P$ and logarithmic divergences in correlation functions. This holds even in the case of arbitrary $\mathbb{Z}_{n}$ twists. Thus, let $\mu_{\lambda}$ denote the field which simulates a branch point of ramification number $n$. In this case, the branch point vertex operator $\Phi_{\mathbf{q}}$ consists out of $n$ copies of fields $\Phi_{q_{k}}$ with the $q_{k}$ given by Eq. 8 . Looking at just one of these copies, $\mu_{\lambda}$ is one of these fields $\Phi_{q_{k}}$ for a given $k \in\{0, \ldots, n-1\}$.

We now come to a subtle point concerning the contours, along which the screening currents are integrated. In our geometrical setting, we would like to choose contours which are non-trivial elements of the homology. In fact, it is possible to choose such a contour, if the two fields which constitute the insertions around which the contour is to be taken, lead to something local with respect to the screening current. The typical situation in ordinary conformal field is different, however. To perform a screening integration, one usually has to take a Pochhammer double loop integral. Now, we observe the following: If a twist field $\mu_{\lambda}$ represents an, say, $\ell$-fold ramification around a branch point, when this point is encircled counter-clockwise, then the twist field $\mu_{\lambda^{*}}$ with $\lambda^{*}=1-\lambda \equiv-\lambda \bmod 1$ represents an $\ell$-fold ramification backwards, if its insertion point is encircled counter-clockwise. This is the situation just mentioned, a pair of two twists $\mu_{\lambda}$ and $\mu_{\lambda^{*}}$, which together yield something local with respect to a simple homology cycle encircling both. A pair of twists $\mu_{\lambda}$ and $\mu_{\lambda^{\prime}}$ with $\lambda^{\prime} \neq 1-\lambda$ mod 1 cannot be encircled by a simple homology cycle, since the single loop cannot close. We depict the two different situations below, for the sake of simplicity in the case of $\mathbb{Z}_{3}$ twists.
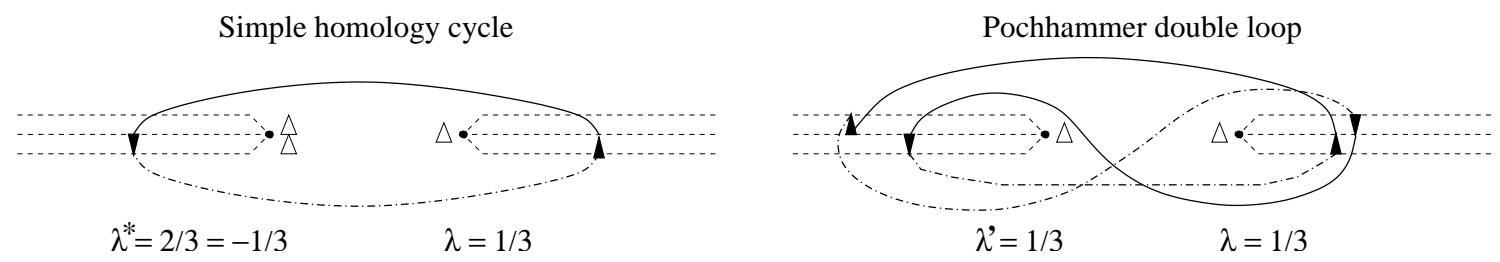

In left situation, we have the possibility to take as screening contour a simple homology cycle, since the second twist with $\lambda^{*}=2 / 3$ undoes the effect of the first with $\lambda=1 / 3$ such that the contour ends on the same sheet where it started. In the right situation, this is not possible, since the second twist cannot undo the effect of the first, since $\lambda^{\prime}=\lambda=1 / 3$ such that $\lambda^{\prime}+\lambda \neq 1$. A more careful consideration shows that a single loop integration is possible whenever $\lambda^{\prime}+\lambda \in \mathbb{Z}$.

Why does this lead to logarithms? Now, the primary fields $\mu_{\lambda}$ are actually superpositions of chiral vertex operators. In the simplest case, the $\mathbb{Z}_{2}$ case, the field $\mu_{1 / 2}$ is actually given by the superposition $\mu_{1 / 2}=\mathcal{D}_{1 / 2, q}^{q+1 / 2} V_{1 / 2, q}^{q+1 / 2}(\cdot, z)+\mathcal{D}_{1 / 2, q}^{q-1 / 2} V_{1 / 2, q}^{q-1 / 2}(\cdot, z)$ where we have that $V_{1 / 2, q}^{q+1 / 2}(\cdot, z)$ is basically the unscreened vertex operator $V_{1 / 2}(z)=\exp \left(\mathrm{i} \frac{1}{2} \varphi(z)\right)$, while $\left.V_{1 / 2, q}^{q-1 / 2}(\cdot, z)=\oint \mathrm{d} z^{\prime} J_{(} z^{\prime}\right) V_{1 / 2}(z)$. This reflects the fact that the field $\mu_{1 / 2}$ is degenerate of level two such that the fusion rules are simply $\left[\mu_{1 / 2}\right] \times\left[\Phi_{q}\right]=\left[\Phi_{q+1 / 2}\right]+\left[\Phi_{q-1 / 2}\right]$ for any primary field $\Phi_{q}$. Let us now study the effect of an operator product expansion of two such fields $\mu_{1 / 2}$. Geometrically, this means that we let two branch points run into each other. The unscreened part of the primary fields $\mu_{1 / 2}$ will simply add up,

$$
V_{1 / 2,1 / 2}^{1}\left(\left|\mu_{1 / 2}\right\rangle, z\right) \quad: \quad \mu_{1 / 2}(z) \mu_{1 / 2}(w) \sim(z-w)^{1 / 4} P(w)
$$


which geometrically means that the two branch points run into each other to form a puncture or marked point on the Riemann sphere. The screened part, however, leads to two different possibilities depending on the choice of the screening contour. If the screening contour encircles the resulting puncture at, say, coordinate $z$, all what happens is that we take the residue of the pole at $z$. The net effect is a constant such that in this situation, the screened part results simply in $Q_{-} P(z)=$ $\oint z^{-1} \mathbb{I}=\mathbb{I}$, the identity operator. On the other hand, if the contour is chosen such that it gets pinched between the two branch points, a single cut survives. Let us assume without loss of generality that the puncture is created by letting a branch point at $z$ run into a branch point at zero. The pinching of the contour leads to an divergence for $z \rightarrow 0$ which reflects in its behavior the appearance of the cut. The integration around the pole cannot be performed to simply yield a residue. Instead, the defect of doing so depends on how close the two branch points get when pinching the contour. Since the contour cannot close, the result must be given by the indefinite integral of $1 / u$, which is $\int^{(z-w)} u^{-1}=\log (z-w)$.
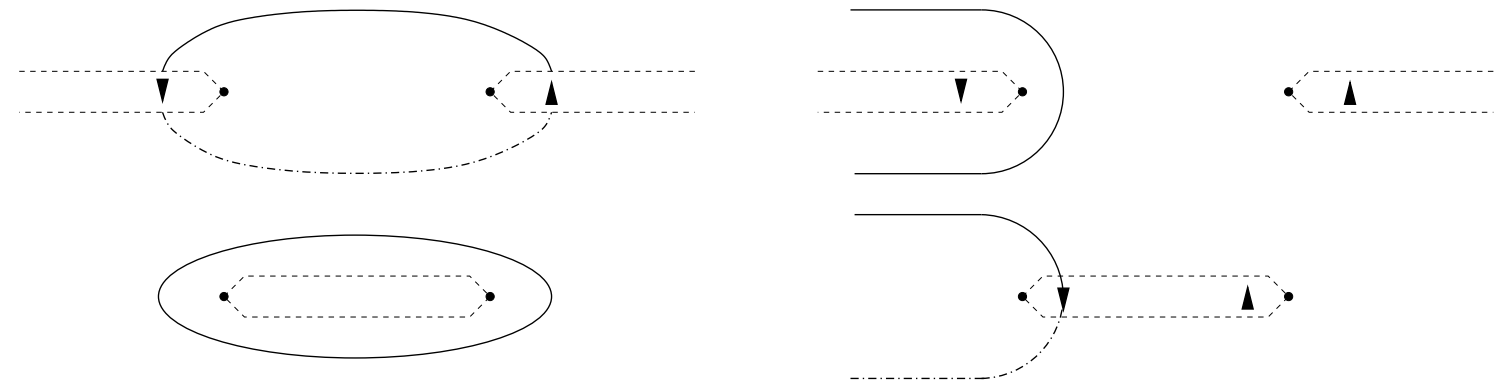

Integration around a puncture/pole

$Q\lrcorner P(z)=\mathbb{I}$

Pinched cycle, carrying a single cut
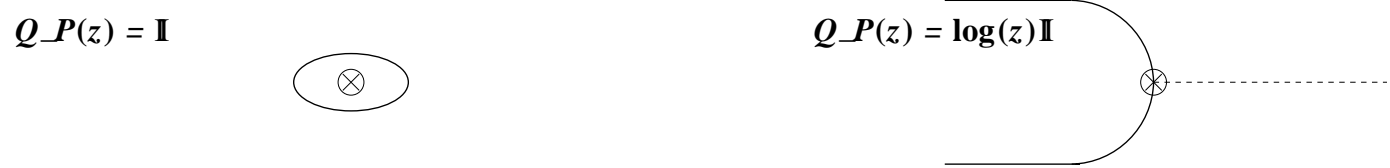

A similar argument holds for arbitrary twists $\mu_{\lambda}$ in the case where the pinching is done with $\mu_{\lambda^{*}}$ resulting in the indefinite integral of $z^{-\lambda-\lambda^{*}}=z^{-1}$, while a pinching of a contour between $\mu_{\lambda}$ and $\mu_{\lambda^{\prime}}$ for $\lambda^{\prime} \neq \lambda^{*}$ does not lead to a logarithm since the indefinite integral now is $\int z^{-\lambda-\lambda^{\prime}}=-\frac{1}{\lambda+\lambda^{\prime}-1} z^{1-\lambda-\lambda^{\prime}}$. Furthermore, the primary field $P$ is again a superposition of chiral vertex operators. Since it is degenerate of level three, the superposition contains three terms with zero, one or two screening integrations attached, respectively. Again, integer order poles may arise leading to logarithms, if one contour is pinched between two of the puncture operators. Indeed, the integral one has actually to perform can be brought into the form

$$
\int^{2 \epsilon} \mathrm{d} u \frac{1}{(u-\epsilon)^{\lambda}(u+\epsilon)^{\lambda^{\prime}}} \sim \int^{2 \epsilon} \mathrm{d} u\left(\frac{1}{u^{\lambda+\lambda^{\prime}}}+\frac{\lambda-\lambda^{\prime}}{u^{\lambda+\lambda^{\prime}+1}} \epsilon+\mathcal{O}\left(\frac{\epsilon^{2}}{u^{\lambda+\lambda^{\prime}+2}}\right)\right),
$$

which develops a simple pole precisely for $\lambda^{\prime}=\lambda^{*}=1-\lambda$, yielding then a logarithmic divergence $\log (2 \epsilon)$. Note that, would we have used a Pochhammer double loop, the logarithmic divergence would appear twice, but with opposite signs due to the fact that each loop comes with both orientations. It is therefore crucial that we use homology cycles as the contours for screening current integrations.

As a demonstration, let us compute the possible conformal blocks of the four-point functions $\left\langle\mu_{1 / 2}(\infty) \mu_{1 / 2}(1) \mu_{1 / 2}(x) \mu_{1 / 2}(0)\right\rangle$. First of all, we have basically two inequivalent ways to join the two pairs of branch points by cuts. Secondly, the homology for the torus is spanned by two elements, $\alpha$ and $\beta$. We have depicted canonical choices for the homology cycles $\alpha$ and $\beta$ for both configurations 
for the branch cuts.
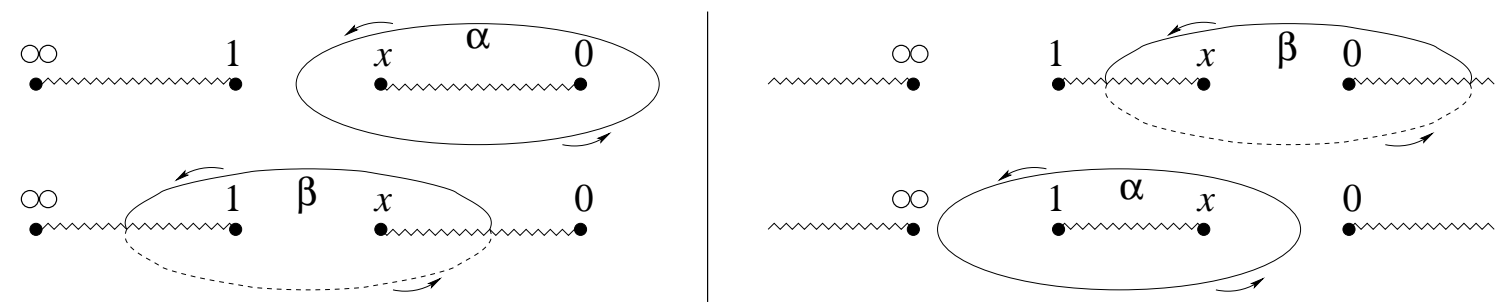

We now insert the OPE for $|x| \ll 1$ such that essentially try to reduce the four-point functions to a three-point function. In the left configuration, the screened vertex operator at $x$ leads after insertion of the OPE to

$$
V_{1 / 2,1 / 2}^{0}\left(\left|\mu_{1 / 2}\right\rangle, x\right) \quad: \quad \mu_{1 / 2}(x) \mu_{1 / 2}(0) \sim x^{1 / 4} \oint_{\alpha} \mathrm{d} u u^{-1} \mathbb{I} \sim x^{1 / 4} \mathbb{I}
$$

for the homology cycle $\alpha$, and for the $\beta$ cycle, the pinching leads to the result

$$
\begin{aligned}
V_{1 / 2,1 / 2}^{0}\left(\left|\mu_{1 / 2}\right\rangle, x\right): \mu_{1 / 2}(x) \mu_{1 / 2}(0) & \sim x^{1 / 4} \oint_{\beta} \mathrm{d} u u^{-1} \mu_{1 / 2}(x) \mu_{1 / 2}(0) \\
& \sim x^{1 / 4} \int^{x} \mathrm{~d} u u^{-1} \mathbb{I}=x^{1 / 4} \log (x) \mathbb{I} .
\end{aligned}
$$

Note that there can be only an even number of pinchings. Thus, logarithms as the one in the last OPE will show up in correlation functions always as pairs. Since the logarithmic divergence is also dependent on the orientation of the contour, any contour pinched twice will lead to the difference of two logarithms.

Therefore, contracting further for the left configuration and the $\alpha$ cycle leads to

$$
\begin{aligned}
\langle\mu(\infty) \mu(1) \mu(x) \mu(0)\rangle_{\alpha} & \sim x^{1 / 4}\left\langle\mu(\infty) \mu(1)\left(Q_{-} P(0)\right\rangle \sim x^{1 / 4}\langle\mu(\infty) \mu(1) \mathbb{I}\rangle\right. \\
& \sim x^{1 / 4}\langle\mu(\infty) \mu(0)\rangle \sim x^{1 / 4}\langle P(0)\rangle=x^{1 / 4}
\end{aligned}
$$

to leading order, where no logarithms show up, since the contour integration can be performed in a region where no pinching occurs. All operators here are to be understood as unscreened vertex operators, since the screening charge has been explicitly inserted. Since there is only one screening allowed due to charge balance, the OPE inserted in the last line yields only one term. Note that $\langle\mathbb{I}\rangle=0$ and $\langle P\rangle=1$. In case of the $\beta$ cycle, we obtain to leading order

$$
\begin{aligned}
\langle\mu(\infty) \mu(1) \mu(x) \mu(0)\rangle_{\beta} & \sim x^{1 / 4}\left\langle\mu(\infty)\left[\mu(1) Q_{-}\left|{ }_{x} P(0)+Q_{-}\right|_{1} P(x) \mu(0)\right]\right\rangle \\
& \sim x^{1 / 4}\langle\mu(\infty)[\mu(1) \log (x) \mathbb{I}(0)-\log (1-x) \mathbb{I}(x) \mu(0)]\rangle \\
& \sim x^{1 / 4}(\log (x)-\log (1-x))\langle\mu(\infty) \mu(0)\rangle \\
& \sim x^{1 / 4}\left(\log (x)-\log (1-x)\langle P(0)\rangle=x^{1 / 4} \log \left(\frac{x}{1-x}\right) .\right.
\end{aligned}
$$

Here, we have tried to indicate, where the screening contour gets pinched. Note that in case of the $\beta$ cycle, two pinchings occur and a relative sign must be taken into account. To leading order, this yields the well-known results $x^{1 / 4} \log (x)$ for $|x| \ll 1$. Of course, we have made use of translational invariance of the correlators to arrive at the second-last line.

The right configuration can be inferred in a completely analogous way. However, it is equivalent to the left configuration if the region $|1-x| \ll 1$ is considered instead. This essentially exchanges the role of the homology cycles $\alpha$ and $\beta$. We thus see that there are two inequivalent conformal blocks in one-to-one correspondence to the basis of homology cycles. The choice of the screening contour implies the choice of the correct screened vertex operators which build up the primary fields, i.e. the choice of internal channel in the OPE. 
As a last remark, we note that the logarithmic divergence also causes the resulting representation $[P]$ to be indecomposable. This can be seen from the fact that $L_{0}$ is the generator of both dilatations, and rotations. The latter, in turn, generate monodromy transformations. Since $\log (x)$ is multivalued, we see that for a pinched contour, we must have $L_{0} Q_{-} P(x)=Q_{-} P(x)+\mathbb{I}$ which is precisely the statement that $L_{0}|P\rangle=|0\rangle$ forms a Jordan cell.

\subsection{Holomorphic Differentials}

Now, we have all the important data to start expressing differential forms on hyperelliptic curves by correlation functions of the $c=-2$ theory. To demonstrate this, we start with a very simple example, namely the canonical holomorphic differentials $\omega_{n}=z^{n} y^{-1} \mathrm{~d} z$ with $n=0, \ldots, g-1$, and $y$ given by (14). These forms are also called Abelian forms of the first kind. We claim that the holomorphic differentials are simply represented by

$$
\omega_{n}=: J(0)^{n}: \prod_{k=1}^{2 g+2} \mu\left(e_{k}\right) J_{-}(z)=\Phi_{-n}(0) \prod_{k=1}^{2 g+2} \Phi_{1 / 2}\left(e_{k}\right) J_{-}(z),
$$

up to a contribution : $\left(\phi^{(j=0)}(\infty)\right)^{n+1-g}$ : of zero mode absorbing fields.

By this, we mean the following: First, the normal ordered product of $n$ currents projects down to the primary field of charge $q=n$ and conformal weight $h_{n+1,1}=n(n+1) / 2$. Next, integrating $\omega_{n}$ along a cycle of the hyperelliptic curve simply results in integrating the screening current along this cycle, i.e.

$$
\oint_{\alpha} \omega_{n}=\left.\Phi_{-n}(0) \prod_{k=1}^{2 g+2} \Phi_{1 / 2}\left(e_{k}\right) Q_{-}\right|_{\alpha},
$$

where $Q_{-}$denotes the screening charge operator $Q_{-}=\oint J_{-}(z)$. This results just in the nonfree part of the correlation function $\left\langle\Phi_{-n}(0) \prod_{k=1}^{2 g+2} \Phi_{1 / 2}\left(e_{k}\right)\right\rangle_{c=-2}$, actually in a certain linear combination with integer coefficients of a basis of conformal blocks of this correlation function, depending on the cycle $\alpha$. Choosing $\alpha$ as an element of a basis of cycles, and keeping in mind equation (23), we finally find

$$
\oint_{\alpha} \omega_{n}=\left\langle\left\langle: P^{n+1-g}(\infty): \Phi_{-n}(0) \prod_{k=1}^{2 g+2} \Phi_{1 / 2}\left(e_{k}\right)\right\rangle\right\rangle_{(\alpha)},
$$

where we define the basis of conformal blocks, labeled by $\alpha$, to be in correspondence with the chosen basis of cycles. Of course, $: P^{m}(\infty)$ : absorbs any zero modes and could equally been written as : $\left(\phi^{(j=0)}(\infty)\right)^{m}$ :, and it is always implicitly understood that the projection of the normal ordered product onto the primary field $\Phi_{m}(\infty)$ is taken.

It is now worth noting that the primary field $\mu=\Phi_{1 / 2} \equiv \Psi_{1,2}$, which creates (half of) the branch cuts, actually is a degenerate field of level 2. It follows that the elements of the period matrix satisfy certain partial differential equations of second order (as well as equations of first order induced by the conformal Ward identities). Reexpressing these equations in the moduli of the hyperelliptic curve (instead of its branch points) relates them to the Picard-Fuchs equations for the periods.

Clearly, more general forms can be considered, and the most general Abelian differential can be brought into the form (up to zero mode absorbing fields)

$$
\begin{aligned}
\omega & =\frac{\prod_{i=1}^{M}\left(z-z_{i}\right)}{\prod_{k=1}^{2 g+2} \sqrt{z-e_{k}} \prod_{j=1}^{N}\left(z-p_{j}\right)} \mathrm{d} z \\
& =\prod_{i=1}^{M} \Phi_{-1}\left(z_{i}\right) \prod_{k=1}^{2 g+2} \Phi_{1 / 2}\left(e_{k}\right) \prod_{j=1}^{N} \Phi_{1}\left(p_{j}\right) J_{-}(z),
\end{aligned}
$$


where the $z_{i}$ and $p_{j}$ do not need to be all disjunct. In this latter case, it is understood that products of fields at the same point are normal ordered. Also, we do not exclude the case that not all the $z_{i}$ are different from the $e_{k}$. Since $P=\Phi_{1} \equiv \Psi_{1,3}$ is a degenerate primary field of level 3 , we immediately see that in the case of meromorphic differentials with poles not at infinity, the periods of such a differential also must satisfy partial differential equations of third order. This is reflected in the fact that the Picard-Fuchs equations for Seiberg-Witten models with massive hypermultiplets are of third order. However, as long as there is only one pole, the periods are still uniquely determined by second order equations. Such forms are related to Abelian forms of the second kind. In fact, since all $n$-point functions of a two-dimensional conformal field theory are in principle determined by its 2-, 3- and 4-point functions, an Abelian differential of the third kind, written as an $n$-point function, can be expressed solely with 4-point functions having at least one field degenerate of level 2 in them.

To effectively compute a general Abelian form which also has zeroes, it is convenient to adopt the usual conformal field theory point of view: Writing the form as

$$
\omega=\prod_{i=1}^{K}\left(z-a_{i}\right)^{r_{i}},
$$

the $r_{i}$ would be restricted to be in $\{-1 / 2,+1 / 2,-1,+1\}$. For the correlation functions, it is better to keep the $r_{i}$ as variable, compute the screening charge integral in a region of the set of exponents $r_{i}$ where everything converges, and then perform an analytic continuation to the correct values of the $r_{i}$. This procedure is particularly helpful for the case that $\omega$ has zeroes. Instead of performing an integration along a path that joins two of these zeroes, we might introduce new cycles which go around two of the zeroes, and perform the calculation as if all the $r_{i}$ were negative. This usually leads to generalized hypergeometric or hyperelliptic integrals within a region of parameter space there the integrals are well-defined and converge. Other regions of the parameter space $\left\{r_{i}\right\}$ can be reached by analytic continuation.

\section{Seiberg-Witten Solutions of Supersymmetric Four-Dimen- sional Yang-Mills Theories}

In a much celebrated work [69, Seiberg and Witten found an exact solution to $N=2$ supersymmetric four-dimensional Yang-Mill theory with gauge group $S U(2)$. This paper initiated a whole new, tree sized, branch of research leading to a vast set of exactly solvable Yang-Mills theories in various dimensions and with various degrees of supersymmetry. For some basic or introductory works see, for example, [56, 45, 2, 40, 70, 68, and references therein. Of particular interest for these solutions is the understanding of the moduli space of vacua, which in many cases turns out to be a hyperelliptic Riemann surface. In particular, simply-laced Lie groups lead to spectral curves which are hyperelliptic.

The BPS spectrum of such a model is entirely determined by the periods of a special meromorphic 1-differential on this Riemann surface, the famous Seiberg-Witten differential $\lambda_{\mathrm{SW}}$. A general hyperelliptic Riemann surface can be described in terms of two variables $w, Z$ in the polynomial form

$$
w^{2}+2 A(Z) w+B(Z)=0
$$

with $A(Z), B(Z) \in \mathbb{C}[Z]$. After a simple coordinate transformation in $y=w-A(Z)$, this takes on the more familiar form $y^{2}=A(Z)^{2}-B(Z)$. But we might also write the hyperelliptic curve in terms of a rational map if we divide the defining equation (38) by $A(Z)^{2}$ and put $\tilde{w}=w / A(Z)+1$ to arrive at the representation

$$
(1-\tilde{w})(1+\tilde{w})=\frac{B(Z)}{A(Z)^{2}} .
$$

This form is very appropriate in the frame of Seiberg-Witten models, since the Seiberg-Witten differential can be read off directly: The rational map $R(Z)=B(Z) / A(Z)^{2}$ is singular at the zeroes of $B(Z)$ and $A(Z)$, and is degenerate whenever its Wronskian $W(R) \equiv W\left(A(Z)^{2}, B(Z)\right)=$ 
$\left(\partial_{Z} A(Z)^{2}\right) B(Z)-A(Z)^{2}\left(\partial_{Z} B(Z)\right)$ vanishes. This is precisely the information encoded in $\lambda_{\mathrm{SW}}$ which for arbitrary hyperelliptic curves, given by a rational map $R(Z)=B(Z) / A(Z)^{2}$, can be expressed as

$$
\lambda_{\mathrm{SW}}=\frac{Z}{2 \pi \mathrm{i}} \mathrm{d}\left(\log \frac{1-\tilde{w}}{1+\tilde{w}}\right)=\frac{1}{2 \pi \mathrm{i}} \mathrm{d}(\log R(Z)) \frac{Z}{\tilde{w}}=\frac{1}{2 \pi \mathrm{i}} \frac{W\left(A(Z)^{2}, B(Z)\right)}{A(Z) B(Z)} \frac{Z \mathrm{~d} Z}{y} .
$$

Note that the fact that the denominator polynomial is a square guarantees the curve to be hyperelliptic. It is this local form of the Seiberg-Witten differential which serves as a metric $\mathrm{d} s^{2}=\left|\lambda_{\mathrm{SW}}\right|^{2}$ on the Riemann surface. And it is this local form which arises as the tension of self-dual strings coming from 3-branes in type II string theory compactifications on Calabi-Yau threefolds. ${ }^{1}$

Let us, for the sake of simplicity, concentrate on $N=2 S U\left(N_{c}\right)$ Yang-Mills theory with $N_{f}$ massive hypermultiplets. Then, the hyperelliptic curve $y^{2}=A(x)^{2}-B(x)$ takes the form

$$
y^{2}=\left(x^{N_{c}}-\sum_{k=2}^{N_{c}} s_{k} x^{N_{c}-k}\right)^{2}-\Lambda^{2 N_{c}-N_{f}} \prod_{i=1}^{N_{f}}\left(x-m_{i}\right)=\prod_{j=1}^{2 N_{c}}\left(x-e_{j}\right),
$$

where we have absorbed any dependency of $A(x)=\prod_{k=1}^{N_{c}}\left(x-a_{k}\right)$ on the $m_{i}$, which is the case for $N_{f}>N_{c}$, in a redefinition of the $a_{k}$ or $s_{k}$ respectively. Then, the Seiberg-Witten differential takes the general form

$$
\lambda_{\mathrm{SW}}\left(S U\left(N_{c}\right)\right)=\frac{1}{2 \pi \mathrm{i}} \frac{x \prod_{l=1}^{N_{c}+N_{f}-1}\left(x-z_{l}\right)}{\prod_{j=1}^{2 N_{c}} \sqrt{x-e_{j}} \prod_{i=1}^{N_{f}}\left(x-m_{i}\right)} \mathrm{d} x,
$$

where the $z_{l}$ denote the zeroes of $2 A(x)^{\prime} B(x)-A(x) B(x)^{\prime}$. As a result, the total order of the general Seiberg-Witten form (40) vanishes, $\left(1+N_{c}+N_{f}-1\right) \cdot(1)+\left(2 N_{c}\right) \cdot\left(-\frac{1}{2}\right)+\left(N_{f}\right) \cdot(-1)=0$, meaning that the charge balance for the corresponding primary fields is identical zero. Hence, the denominator in (23) is just the free part (22). The periods of this differential along a basis of cycles which can be chosen in such a way that they encircle pairs $\left(e_{i}, e_{j}\right)$ are then given by the conformal blocks (including a factor $J_{+}(\infty) \sim: P(\infty)^{2}: \sim \Phi_{2}(\infty)$ for the double pole of the Seiberg-Witten differential at infinity)

$$
\begin{aligned}
a_{\left(e_{i}, e_{j}\right)} & =\oint_{C_{\left(e_{i}, e_{j}\right)}} \lambda_{\mathrm{SW}}\left(S U\left(N_{c}\right)\right) \\
& =\frac{1}{2 \pi \mathrm{i}}\left\langle\left\langle\prod_{j=1}^{2 N_{c}} \mu\left(e_{j}\right) \prod_{i=1}^{N_{f}} P\left(m_{i}\right) \prod_{l=0}^{N_{c}+N_{f}-1} J\left(z_{l}\right): P(\infty)^{2}:\right\rangle_{\left(e_{i}, e_{j}\right)},\right.
\end{aligned}
$$

where we have defined $z_{0}=0$. However, once we live in the CFT picture, we may also define "periods" which run along cycles encircling two zeroes of $\lambda_{\mathrm{SW}}$. We will investigate this in more detail with the help of an explicit example.

But before we do so, we would like to make some more general remarks. First of all, the Kähler potential for the metric on the field space of $N=2$ supersymmetric Yang-Mills theories, $K\left(a_{i}, \bar{a}_{j}\right)=\frac{\mathrm{i}}{2}\left(\left(\partial_{\bar{a}_{j}} \overline{\mathcal{F}}\right) a_{i}-\left(\partial_{a_{i}} \mathcal{F}\right) \bar{a}_{j}\right)$ with $\mathcal{F}\left(\left\{a_{i}\right\}\right)$ the holomorphic prepotential, precisely resembles the single valued conformally invariant combinations of left and right chiral conformal blocks,

$$
K_{i}^{j}=\frac{\mathrm{i}}{2}\left(a_{i} \bar{a}_{D}^{j}-\bar{a}_{i} a_{D}^{j}\right) .
$$

This unusual off-diagonal combination of conformal blocks is a typical feature of logarithmic CFTs. The full single-valued correlation function is nothing else than $\left\langle\left\langle\left|\lambda_{\mathrm{SW}}\right|^{2}\right\rangle\right\rangle=\operatorname{tr} K$. Since the periods

\footnotetext{
${ }^{1}$ This derivation of the Seiberg-Witten differential is equivalent to the one from integrable Toda systems with spectral curve $z+1 / z+r(t)=z+1 / z+2 A(t) / \sqrt{B(t)}=0$, where $\lambda_{\mathrm{SW}}=t \mathrm{~d}(\log z)$ is nothing else than the Hamilton-Jacobi function of the underlying integrable hierarchy. However, the price paid for this very simple form of $\lambda_{\mathrm{SW}}$ is that $r(t)$ is now only a fractional rational map.
} 
of the holomorphic 1-forms are also expressed in terms of conformal blocks, similar results hold for the metric on field space itself,

$$
(\mathrm{d} s)^{2}=\Im \mathfrak{m} \mathrm{d} a_{D}^{i} \mathrm{~d} \bar{a}_{i}
$$

when the exterior derivatives are expressed as derivatives with respect to the moduli $u_{k}$ or $s_{k}$. Therefore, the meromorphic 1 -form $\lambda_{\mathrm{SW}}$ is a generating differential, and the $S L(2, \mathbb{C})$ invariance of the metric is nothing else than the conformal invariance of the correlation functions of our CFT. Note that again $(\mathrm{d} s)^{2}$ is identical to the full single-valued correlation function combined out of the conformal blocks. More generally, we can define for an arbitrary Abelian differential $\Omega$ the analogue of the Kähler potential, $\mathcal{K}(\Omega)_{i}{ }^{j}=\frac{i}{2}\left(\oint_{\alpha_{i}} \Omega \oint_{\beta^{j}} \bar{\Omega}-\oint_{\alpha_{i}} \bar{\Omega} \oint_{\beta^{j}} \Omega\right)$. Moreover, duality in $N=2$ supersymmetric Yang-Mills theories is nothing else than crossing symmetry of 4-point (and higherpoint) functions. Finally, the coupling constants are given in terms of the period matrix. On a genus $g$ Riemann surface, the period matrix is defined for a canonical symplectic basis of cycles $\left\{\alpha_{i}, \beta^{i}\right\}_{1 \leq i \leq g}$ and a basis of the holomorphic 1-differentials $\left\{\omega_{i}\right\}_{1 \leq i \leq g}$ by the $(g, 2 g)$ matrix

$$
\left(\Pi_{D j}{ }^{i}, \Pi_{i j}\right)=\left(\oint_{\beta^{i}} \omega_{j}, \oint_{\alpha_{i}} \omega_{j}\right)
$$

So, the coupling constants are simply $\tau^{i k}=\left(\Pi^{-1} \Pi_{D}\right)^{i k}$, and thus expressed in terms of correlation functions. For completeness, we also mention that the first and second Riemann bilinear relation, $\tau-(\tau)^{t}=0$ and $\Im \mathfrak{m}(\tau)>0$, are immediate consequences.

\subsection{Periods of the Seiberg-Witten Differential}

We will look at the simplest example first, the Seiberg-Witten model with gauge group $S U(2)$. The resulting Riemann surface is then simply of genus one, a torus.

Let us start with a warm up by calculating the periods of the only holomorphic one-form for the torus. The torus in question is given by $y^{2}=\left(x^{2}-u\right)^{2}-\Lambda^{4}$ with the four branch points $e_{1}=\sqrt{u-\Lambda^{2}}, e_{2}=-\sqrt{u+\Lambda^{2}}, e_{3}=-\sqrt{u-\Lambda^{2}}, e_{4}=\sqrt{u+\Lambda^{2}}$. The standard periods of the holomorphic form $\mathrm{d} x / y$ are easily computed (where the normalization has been fixed to be in accordance with the asymptotic behavior of $a$ and $a_{D}$ in the weak coupling region). With $\xi=$ $1 / M\left(e_{4}\right)=\frac{\left(e_{1}-e_{4}\right)\left(e_{3}-e_{2}\right)}{\left(e_{2}-e_{1}\right)\left(e_{4}-e_{3}\right)}$ the inverse crossing ratio, $\xi=\left(u-\sqrt{u^{2}-\Lambda^{4}}\right) /\left(u+\sqrt{u^{2}-\Lambda^{4}}\right)$, we have

$$
\begin{aligned}
\pi_{1}=\frac{\partial a}{\partial u} & =\frac{\sqrt{2}}{2 \pi} \int_{e_{2}}^{e_{3}} \frac{\mathrm{d} x}{y}=\frac{\sqrt{2}}{2 \pi}\left\langle\mu\left(e_{1}\right) \mu\left(e_{2}\right) \mu\left(e_{3}\right) \mu\left(e_{4}\right)\right\rangle_{\left(e_{2}, e_{3}\right)} \\
& =\frac{\sqrt{2}}{2 \pi}\left(e_{3}-e_{2}\right)^{-\frac{1}{2}}\left(e_{4}-e_{1}\right)^{-\frac{1}{2}}\left\langle\mu(\infty) \mu(1) \mu(0) \mu\left(M\left(e_{4}\right)\right)\right\rangle(0,1) \\
& =\frac{\sqrt{2}}{2}\left(e_{2}-e_{1}\right)^{-\frac{1}{2}}\left(e_{4}-e_{3}\right)^{-\frac{1}{2}}{ }_{2} F_{1}\left(\frac{1}{2}, \frac{1}{2} ; 1 ; \xi\right)
\end{aligned}
$$

The other period is obtained in complete analogy by exchanging $e_{2}$ with $e_{1}$, yielding

$$
\pi_{2}=\frac{\partial a_{D}}{\partial u}=\frac{\sqrt{2}}{2 \pi} \int_{e_{1}}^{e_{3}} \frac{\mathrm{d} x}{y}=\frac{\sqrt{2}}{2}\left(e_{1}-e_{2}\right)^{-\frac{1}{2}}\left(e_{4}-e_{3}\right)^{-\frac{1}{2}}{ }_{2} F_{1}\left(\frac{1}{2}, \frac{1}{2} ; 1 ; 1-\xi\right) .
$$

Here and in the following, (generalized) hypergeometric functions with arguments such as $1-$ $\xi$ are understood as expansions around $1-\xi$ and should be analytically continued to a region around $\xi$. This will result in the desired logarithmic divergences. For example, with the usual Frobenius process we find (the factor $\pi=\Gamma\left(\frac{1}{2}\right)^{2}$ stems from the formula for analytic continuation of hypergeometric functions)

$$
\begin{aligned}
\pi_{2} F_{1}\left(\frac{1}{2}, \frac{1}{2} ; 1 ; 1-\xi\right)= & { }_{2} F_{1}\left(\frac{1}{2}, \frac{1}{2} ; 1 ; \xi\right) \log (\xi)+\left.\sum_{n=0}^{\infty}\left(\frac{\partial}{\partial \varepsilon} \frac{\left(\frac{1}{2}+\varepsilon\right)_{n}\left(\frac{1}{2}+\varepsilon\right)_{n}}{(1+\varepsilon)_{n}(1+\varepsilon)_{n}}\right)\right|_{\varepsilon=0} \xi^{n} \\
= & { }_{2} F_{1}\left(\frac{1}{2}, \frac{1}{2} ; 1 ; \xi\right) \log (\xi) \\
& +\left.\partial_{\varepsilon 3} F_{2}\left(1, \frac{1}{2}+\varepsilon, \frac{1}{2}+\varepsilon ; 1+\varepsilon, 1+\varepsilon ; \xi\right)\right|_{\varepsilon=0} .
\end{aligned}
$$


These results are, of course, well known. Less known might be the fact that for the case without hyper-multiplets, $N_{f}=0$, we can express the periods of the Seiberg-Witten form by the Lauricella function $F_{D}^{(3)}$. In fact,

$$
\begin{aligned}
a(u)= & \frac{\sqrt{2}}{2 \pi} \int_{e_{2}}^{e_{3}} \frac{4 x^{2} \mathrm{~d} x}{y}=\frac{2 \sqrt{2}}{\pi}\left\langle\Phi_{2}(\infty) \mu\left(e_{1}\right) \mu\left(e_{2}\right) \mu\left(e_{3}\right) \mu\left(e_{4}\right) \Phi_{-2}(0)\right\rangle_{\left(e_{2}, e_{3}\right)} \\
= & \frac{2 \sqrt{2}}{\pi} \frac{e_{1}^{2}}{\left(e_{3}-e_{2}\right)^{\frac{1}{2}}\left(e_{4}-e_{1}\right)^{\frac{1}{2}}} \\
& \times\left\langle\mu(\infty) \mu(1) \mu(0) \mu\left(M\left(e_{4}\right)\right) \Phi_{-2}(M(0)) \Phi_{2}(M(\infty))\right\rangle_{(0,1)} \\
= & 2 \sqrt{2} \frac{e_{3}^{2}}{\left(e_{4}-e_{3}\right)^{\frac{1}{2}}\left(e_{2}-e_{1}\right)^{\frac{1}{2}}} F_{D}^{(3)}\left(\frac{1}{2}, \frac{1}{2},-2,2,1 ; \xi, \eta, \varpi\right),
\end{aligned}
$$

with the second inverse cross ratio $\eta=1 / M(0)=\frac{e_{1}\left(e_{2}-e_{3}\right)}{\left(e_{1}-e_{2}\right) e_{3}}$, and $\varpi=1 / M(\infty)=\frac{e_{2}-e_{3}}{e_{2}-e_{1}}$ the inverse of the image of the double pole at infinity (which absorbs the zero modes). The Lauricella $D$ type functions are generalized hypergeometric functions in several variables. We collect all we need about them in Appendix A. For $n=1$, they reduce to the ordinary Gauss hypergeometric functions ${ }_{2} F_{1}\left(a, b_{1} ; c ; x_{1}\right)$, and for $n=2$, they are nothing else than the Appell functions $F_{1}\left(a ; b_{1}, b_{2} ; c ; x_{1}, x_{2}\right)$. A great deal of information on these functions may be found for example in the book [18 by Exton. An important fact is that $F_{D}^{(n)}$ satisfies the following system of partial differential equations of second order:

$$
\begin{aligned}
\mathcal{D}_{j} & =\left(1-x_{j}\right) \sum_{k=1}^{n} x_{k} \frac{\partial^{2}}{\partial x_{k} \partial x_{j}}+\left(c-\left(a+b_{j}+1\right) x_{j}\right) \frac{\partial}{\partial x_{j}}-b_{j} \sum_{\substack{k=1 \\
k \neq j}}^{n} x_{k} \frac{\partial}{\partial x_{k}}-a b_{j}, \\
0 & =\mathcal{D}_{j} F_{D}^{(n)},
\end{aligned}
$$

where $j=1, \ldots, n$. Interestingly, this remains true even in the case that massive hypermultiplets are present $\left(N_{f}>0\right)$, while the Picard-Fuchs equations now are of third order. However, the price paid is an artificially enlarged number of variables. Furthermore, we easily can write down differential equations of second and third order for each field in the correlator which is proportional to $F_{D}^{(n)}$, depending on whether the field is degenerate of level two, e.g. $\mu=\Psi_{1,2}, \Phi_{-1}=\Psi_{2,1}$, or three as $\Phi_{1}=\Psi_{1,3}$ (where we consider the $c=-2$ CFT as the degenerate model with $c=c_{2,1}$ ) according to 3 .

Again, we may obtain the dual period by exchanging $e_{2}$ with $e_{1}$, yielding

$$
a_{D}(u)=2 \sqrt{2} \frac{e_{3}^{2}}{\left(e_{4}-e_{3}\right)^{\frac{1}{2}}\left(e_{1}-e_{2}\right)^{\frac{1}{2}}} F_{D}^{(3)}\left(\frac{1}{2}, \frac{1}{2},-2,2,1 ; 1-\xi, 1-\eta, 1-\varpi\right) .
$$

The two periods given above are by construction the $a_{(\alpha)}$ and $a_{(\beta)}$ periods respectively. In the same way, we find the period integrated between $e_{2}$ and $e_{4}$, which is

$$
a_{(2 \alpha-\beta)}(u)=2 \sqrt{2} \frac{-e_{2}^{2}}{\left(e_{4}-e_{3}\right)^{\frac{1}{2}}\left(e_{1}-e_{2}\right)^{\frac{1}{2}}} F_{D}^{(3)}\left(\frac{1}{2}, \frac{1}{2},-2,2,1 ; 1-\xi, \frac{\xi-1}{\eta-1}, \frac{\xi-1}{\varpi-1}\right) .
$$

It is worth noting that the dependency on three variables is superficial, since all cross ratios are solely functions in the four branch points. Indeed, we have $\xi=\varpi^{2}, \eta=-\varpi$. The inverse crossing ratios have the nice property that they tend to zero for $|u| \gg 1$, e.g. $\xi \sim\left(\frac{1}{2} \frac{\Lambda^{2}}{u}\right)^{2}+O\left(u^{-4}\right)$. Hence, the overall asymptotics of $a(u)$ and $a_{D}(u)$ is entirely determined by the prefactors, which are $a(u) \sim$ $\frac{2 \sqrt{2} e_{3}^{2}}{\sqrt{e_{4}-e_{3}} \sqrt{e_{2}-e_{1}}} \sim \sqrt{2 u}+O\left(u^{-\frac{1}{2}}\right)$ and $a_{D}(u) \sim \frac{\sqrt{2} e_{3}^{2}}{\pi \sqrt{e_{4}-e_{3}} \sqrt{e_{1}-e_{2}}} \log (\xi) \sim \frac{\mathrm{i}}{\pi} \sqrt{2 u} \log (u)+O\left(u^{-\frac{1}{2}} \log (u)\right)$. Expanding $a(u)$ as a power series in $1 / u$ yields the familiar result

$$
\begin{aligned}
a(u) & =\sqrt{2 u}\left[1-\frac{1}{16} \frac{\Lambda^{4}}{u^{2}}-\frac{15}{1024} \frac{\Lambda^{8}}{u^{4}}-\frac{105}{16384} \frac{\Lambda^{12}}{u^{6}}-\frac{15015}{4194304} \frac{\Lambda^{16}}{u^{8}}+O\left(u^{-10}\right)\right] \\
& =\sqrt{2} \sqrt{u+\Lambda^{2}}{ }_{2} F_{1}\left(-\frac{1}{2}, \frac{1}{2}, 1 ; \frac{2 \Lambda^{2}}{u+\Lambda^{2}}\right) .
\end{aligned}
$$




\subsection{Asymptotics and OPEs}

The strength of the CFT picture becomes apparent when asymptotic regions of the moduli space are to be explored. Then, OPE and fusion rules provide easy and suggestive tools. For example, the asymptotics of $a(u)$ and $a_{D}(u)$ follow directly from the OPE of the field $\mu$ as discussed in the preceding section. The logarithmic partners of primary fields appear precisely, if the contour of the screening charge integration gets pinched between the two fields whose OPE is inserted. Thus, the choice of contour together with the choice of internal channels (due to inserted OPEs) determines which term of the OPE is picked, either $\mu(z) \mu(0) \sim z^{1 / 4}(\mathbb{I}(0)+\ldots)$ or $\mu(z) \mu(0) \sim z^{1 / 4}(P(0)-2 \log (z) \mathbb{I}(0)+\ldots)$. So, when expanded in $\xi$, both periods, $a(u)$ and $a_{D}(u)$ have asymptotics according to inserting the OPEs $\mu\left(e_{2}\right) \mu\left(e_{3}\right)$ and $\mu\left(e_{1}\right) \mu\left(e_{4}\right)$. Keeping in mind (23) when inserting an OPE, we find with $e_{i j}=e_{i}-e_{j}$

$$
\begin{aligned}
a(u) & \sim\left[e_{12} e_{13} e_{42} e_{43}\right]^{-1 / 4} \frac{e_{1} e_{2}}{e_{3} e_{4}}\left[e_{34}\left\langle\Phi_{2}(\infty) \mathbb{I}\left(e_{3}\right) P\left(e_{4}\right) \Phi_{-2}(0)\right\rangle+\ldots\right] \\
& \sim\left[e_{12} e_{13} e_{42} e_{43}\right]^{-1 / 4} \frac{e_{1} e_{2} e_{4}}{e_{3}}\left[\left\langle\Phi_{2}(\infty) P\left(e_{4}\right) \Phi_{-2}(0)\right\rangle+\ldots\right] \\
& \sim \sqrt{2 u}+\ldots,
\end{aligned}
$$

where the three-point functions evaluate trivially. Of course, the result is the same if we had chosen the OPEs vice versa such that at the points $e_{3}$ and $e_{4}$ we would have inserted $P\left(e_{3}\right) \mathbb{I}\left(e_{4}\right)$ instead. In a similar fashion, we obtain

$$
\begin{aligned}
a_{D} & (u) \\
& \sim \frac{1}{\mathrm{i} \pi}\left[e_{12} e_{13} e_{42} e_{43}\right]^{-1 / 4} \frac{e_{1} e_{2}}{e_{3} e_{4}}\left[e_{34}\left\langle\Phi_{2}(\infty) P\left(e_{3}\right) P\left(e_{4}\right) \Phi_{-2}(0)\right\rangle+\ldots\right] \\
& \left.\sim \frac{\mathrm{i}}{\pi} \sqrt{2 u} e_{13} e_{42} e_{43}\right]^{-1 / 4} \frac{e_{1} e_{2} e_{4}}{e_{3}}\left[-2 \log (u)+2 \log \left(e_{43}\right)\left\langle\Phi_{2}(\infty) P\left(e_{3}\right) \Phi_{-2}(0)\right\rangle+\ldots\right]
\end{aligned}
$$

Of course, other internal channels can be considered. In particular, we may insert the OPE for $\left|e_{1}-e_{3}\right| \ll 1$ to get the behavior of the periods for the case $u \longrightarrow \Lambda^{2}$. In fact, $a_{D}(u)$ and $a(u)$ exchange their rôle since now the monopole becomes massless. Put differently, duality in SeibergWitten models cooks down to crossing symmetry in our $c=-2$ LCFT. The leading term can be read off from $a_{D}(u)$ above (the OPE factors turn out to be the same up to a braiding phase) to be proportional to $i\left(u-\Lambda^{2}\right) / \sqrt{2 \Lambda^{2}}$. The relative normalization of the logarithmic operator $\Lambda_{1}$ with respect to its primary partner is fixed by considering $a_{D}(u)$ as the analytic continuation of $a(u)$ via crossing symmetry yielding a factor of $(i \pi)^{-1}$.

There is one further BPS state which can become massless, since there is one further zero of the discriminant

$$
\Delta\left(y^{2}(x)\right)=\left(\operatorname{det} \bar{\partial}_{\left(j=\frac{1}{2}\right)}\right)^{8}=\left(\left\langle\prod_{i=1}^{2 g+2} \Phi_{1 / 2}\left(e_{i}\right)\right\rangle_{c=1}\right)^{8}=\prod_{j<k}\left(e_{j}-e_{k}\right)^{2}
$$

namely $e_{2} \longrightarrow e_{4}$. This is a dyonic state with charge $(q, g)=(-2,1)$, meaning that both, the $\alpha$ cycles as well as the $\beta$ cycle, get pinched in this limit. It follows that both, $a(u)$ as well as $a_{D}(u)$, will receive logarithmic corrections when $u \longrightarrow-\Lambda^{2}$, which is well known to be the case.

Within the CFT picture, higher gauge groups as well as additional (massive) flavors are treated in the same way. Hence, we obtain for the $S U(2)$ case with $N_{f}<4$ hypermultiplets, after simple algebra in the numerator,

$$
\begin{aligned}
\lambda_{\mathrm{SW}}= & \frac{1}{2 \pi \mathrm{i}} \frac{x \mathrm{~d} x}{y \prod_{k=1}^{N_{f}}\left(x-m_{k}\right)} \\
& \times\left(4 x \prod_{k=1}^{N_{f}}\left(x-m_{k}\right)-(x-\sqrt{u})(x+\sqrt{u}) \sum_{k=1}^{N_{f}} \prod_{l \neq k}\left(x-m_{l}\right)\right)
\end{aligned}
$$




$$
=\frac{\mathrm{d} x}{2 \pi \mathrm{i}}\left(\left(4-N_{f}\right) \frac{x^{2}}{y}+N_{f} \frac{u}{y}-\sum_{k=1}^{N_{f}} m_{k}\left(\frac{x^{2}}{y\left(x-m_{k}\right)}-\frac{u}{y\left(x-m_{k}\right)}\right)\right),
$$

such that we immediately can express the periods of the Seiberg-Witten form in 4-point and 5-point functions. Using $\frac{x^{2}}{y\left(x-m_{k}\right)}=\frac{x+m_{k}}{y}+\frac{m_{k}^{2}}{y\left(x-m_{k}\right)}$ to rewrite the last term, we obtain

$$
\begin{aligned}
\oint \lambda_{\mathrm{SW}}= & \frac{1}{2 \pi \mathrm{i}}\left(\left(4-N_{f}\right)\left\langle\Phi_{2}(\infty) \mu\left(e_{1}\right) \mu\left(e_{2}\right) \mu\left(e_{3}\right) \mu\left(e_{4}\right) \Phi_{-2}(0)\right\rangle\right. \\
& +u N_{f}\left\langle\mu\left(e_{1}\right) \mu\left(e_{2}\right) \mu\left(e_{3}\right) \mu\left(e_{4}\right)\right\rangle \\
& -\sum_{k=1}^{N_{f}} m_{k}\left[\left\langle\Phi_{1}(\infty) \mu\left(e_{1}\right) \mu\left(e_{2}\right) \mu\left(e_{3}\right) \mu\left(e_{4}\right) \Phi_{-1}\left(-m_{k}\right)\right\rangle\right. \\
& \left.\left.-\left(u-m_{k}^{2}\right)\left\langle\Phi_{-1}(\infty) \mu\left(e_{1}\right) \mu\left(e_{2}\right) \mu\left(e_{3}\right) \mu\left(e_{4}\right) \Phi_{1}\left(m_{k}\right)\right\rangle\right]\right)
\end{aligned}
$$

as the CFT expression. We recover hence the well know result that for all $m_{k}=0$ the scalar modes have roughly the same form as in the $N_{f}=0$ case. Including the charge balance at infinity, and again using $e_{i j}=e_{i}-e_{j}$, the above results in the following expression $(x(\cdot)=1 / M(\cdot)$ denote the inverse crossing ratios)

$$
\begin{aligned}
\oint \lambda_{\mathrm{SW}} & =\left(\frac{\left(4-N_{f}\right) e_{3}^{2}}{\left(e_{43}\right)^{\frac{1}{2}}\left(e_{21}\right)^{\frac{1}{2}}} F_{D}^{(3)}\left(\frac{1}{2}, \frac{1}{2},-2,2,1 ; x\left(e_{4}\right), x(0), x(\infty)\right)\right. \\
& +\frac{u N_{f}}{\left(e_{21}\right)^{\frac{1}{2}}\left(e_{43}\right)^{\frac{1}{2}}}{ }_{2} F_{1}\left(\frac{1}{2}, \frac{1}{2} ; 1 ; x\left(e_{4}\right)\right) \\
& -\sum_{k=1}^{N_{f}} \frac{m_{k}\left(e_{3}+m_{k}\right)}{\left(e_{21}\right)^{\frac{1}{2}}\left(e_{43}\right)^{\frac{1}{2}}} F_{D}^{(3)}\left(\frac{1}{2}, \frac{1}{2},-1,1,1 ; x\left(e_{4}\right), x\left(-m_{k}\right), x(\infty)\right) \\
& \left.+\sum_{k=1}^{N_{f}} \frac{m_{k}\left(u-m_{k}^{2}\right)}{\left(e_{21}\right)^{\frac{1}{2}}\left(e_{43}\right)^{\frac{1}{2}}\left(e_{3}-m_{k}\right)} F_{D}^{(3)}\left(\frac{1}{2}, \frac{1}{2}, 1,-1,1 ; x\left(e_{4}\right), x\left(m_{k}\right), x(\infty)\right)\right) .
\end{aligned}
$$

Since the $F_{D}^{(3)}$ Lauricella functions have a negative integer as one of the numerator parameters, they can be expanded as polynomials in $F_{1}$ Appell functions, i.e. 5-point functions via

$$
F_{D}^{(3)}\left(a ; b, b^{\prime}, b^{\prime \prime} ; c ; x, y, z\right)=\sum_{m=0}^{\infty} \frac{(a)_{m}\left(b^{\prime}\right)_{m} y^{m}}{(1)_{m}(c)_{m}} F_{1}\left(a+m ; b, b^{\prime \prime} ; c+m ; x, z\right),
$$

since this expansion truncates for $b^{\prime} \in \mathbb{Z}_{-}$. Of course, we could have expressed this from the beginning by only one correlation function proportional to $F_{D}^{\left(2 N_{f}+3\right)}$ of $2 N_{f}+3$ variables, as indicated in (35), which is to be contrasted with the approach taken in [10].

As one further example, we consider $S U(3)$ without hypermultiplets, where $R(Z)=\Lambda^{6} /\left(Z^{3}-\right.$ $u Z+v)^{2}$ such that the resulting hyperelliptic curve has six branch points $e_{i}$ and its metric $\left|\lambda_{\mathrm{SW}}\right|^{2}$ possesses three zeroes $z_{j}$. We get

$$
\begin{gathered}
a \oint_{\gamma} \lambda_{\mathrm{SW}}=2\left\langle\left\langle\Phi_{2}(\infty) \mu\left(e_{1}\right) \ldots \mu\left(e_{6}\right) \Phi_{-1}(-\sqrt{u / 3}) \Phi_{-1}(0) \Phi_{-1}(\sqrt{u / 3})\right\rangle\right\rangle_{(\gamma)} \\
=\prod_{i=1}^{3}\left(\partial_{e_{i}} M\left(e_{i}\right)\right)^{\frac{1}{4}} \prod_{i=4}^{6}\left(\frac{\partial_{e_{i}} M\left(e_{i}\right)}{M\left(e_{i}\right)^{2}}\right)^{\frac{1}{4}} \prod_{j=1}^{3}\left(\frac{\partial_{z_{j}} M\left(z_{j}\right)}{M\left(z_{j}\right)^{2}}\right)^{-\frac{1}{2}} \lim _{z \rightarrow \infty}\left(\frac{z^{2} \partial_{z} M(z)}{M(z)^{2}}\right) \\
\times \quad F_{D}^{(7)}\left(\frac{1}{2}, \frac{1}{2}, \frac{1}{2}, \frac{1}{2},-1,-1,-1,2,1 ;\right. \\
\left.\quad x\left(e_{4}\right), x\left(e_{5}\right), x\left(e_{6}\right), x(0), x(-\sqrt{u / 3}), x(\sqrt{u / 3}), x(\infty)\right)
\end{gathered}
$$


with the last equality valid for $\gamma=\alpha_{1} \equiv C\left(e_{2}, e_{3}\right)$. This Lauricella $D$-system for seven variables provides the complete set of all periods. There exist more compact expressions in the literature for this case, where the Appell function $F_{4}$ is involved [46. However, presenting the solution in this way is more transparent, if we view the moduli space of low-energy effective field theory as created from string- or $M$-theory, e.g. as intersecting $N S-5$ and $D$-4 branes. Then, the branch points $e_{i}$ and mass poles $m_{k}$ are the directly given data - they denote the endpoints of the intersections. It remains to interpret the zeroes of the Seiberg-Witten form within the brane picture, since they appear on equal footing with the other singular points in our CFT approach. Moreover, this approach suggests that BPS states from geodesic integration paths [47, 67, 9] joining two zeroes of $\lambda_{\mathrm{SW}}$ can be described in much the same way as the more familiar BPS states connected to the periods. The zeroes of $\lambda_{\mathrm{SW}}$ correspond to branching points in the fibration of Calabi-Yau threefold compactifications of type II string theory, and the corresponding BPS states are related to 2-branes ending on the 5-brane worldvolume $\mathbb{R}^{4} \times \Sigma$.

Expressing the Seiberg-Witten periods in terms of correlation functions reveals a further complication in exploring the moduli space of low-energy effective field theories. These periods depend only on the moduli $s_{k}$ and perhaps masses $m_{l}$. So, for the $S U(3)$ example above, the periods really depend only on two variables, $u, v$. However, $\lambda_{\mathrm{SW}}$ in its factorized form naturally leads to a 10-point function! The complete set of solutions of the associated Lauricella $F_{D}^{(7)}$ system which covers all of $\mathbb{C}^{7}$ is actually quite large, and exceeds by far the set of periods obtainable from simple paths enclosing two of the singular points (Pochhammer paths). As is demonstrated in [18, one needs in addition at least so-called trefoil loops which are self-intersecting contours dividing the set of singularities into three disjunct groups.

The reason behind all this enrichment is buried in the fact that we are dealing with a Riemann surface together with an associated metric $\lambda_{\mathrm{SW}}$. A detailed analysis of all these features relies on a deeper knowledge of the analytic properties of Lauricella functions and will be carried out in our forthcoming paper [1].

\section{The plane versus the torus}

In this last section, we wish to make more explicit contact between the quantities we computed for the elliptic case $g=1$, i.e. the torus, by computing correlation functions on the plane with four $\mathbb{Z}_{2}$ branch point insertions, and well known results for the torus, parameterized as a lattice spanned by $(1, \tau)$, the modulus of the torus. When we considered the conformal blocks of the four-point functions $\langle\mu(\infty) \mu(1) \mu(x) \mu(0)\rangle$, we essentially looked at the periods of the torus. Now, in canonical normalization, we have that the integral of the unique holomorphic form $\omega$ on the torus is given by

$$
\oint_{\alpha} \omega=1, \quad \oint_{\beta} \omega=\tau .
$$

What does this have to do with our results, which were expressed in terms of hypergeometric functions,

$$
\oint_{\alpha} \omega=[x(1-x)]^{1 / 4}{ }_{2} F_{1}\left(\frac{1}{2}, \frac{1}{2}, 1 ; x\right), \quad \oint_{\beta} \omega=[x(1-x)]^{1 / 4}{ }_{2} F_{1}\left(\frac{1}{2}, \frac{1}{2}, 1 ; 1-x\right) ?
$$

How are the torus amplitudes be related to correlation functions on the plane? Before we can see, how these quantities are indeed related, we first have to briefly review the characters and torus amplitudes.

\subsection{Characters and torus amplitudes}

The theory with $c=-2$ is presumably the best understood LCFT. It is possible to compute characters for all its irreducible representations. Besides the vacuum representation, there are the admissible irreducible representations given by the highest weight states created from the twist fields $\mu_{\lambda}$. Kausch [4] has analyzed all of these for the rational case $n \lambda \equiv 0 \bmod 1$ for an $n \in \mathbb{N}$. 
We will restrict ourselves here to the case $n=2$, i.e. the twist fields which generate hyperelliptic ramifications. The characters of the highest weight representations for $h=-1 / 8$ and $h=3 / 8$ are given by

$$
\chi_{-1 / 8}(q)=\frac{\theta_{0,2}(q)}{\eta(q)}, \quad \chi_{3 / 8}(q)=\frac{\theta_{2,2}(q)}{\eta(q)},
$$

where the Jacobi-Riemann theta-functions and the Dedekind eta-function are defines as

$$
\theta_{\lambda, k}(q)=\sum_{n \in \mathbb{Z}} q^{(2 k n+\lambda)^{2} / 4 k}, \quad \eta(q)=q^{1 / 24} \prod_{n=1}^{\infty}\left(1-q^{n}\right) .
$$

There are two more irreducible representations, namely for $h=0$ and $h=1$, with characters

$$
\chi_{0}(q)=\frac{1}{2 \eta(q)}\left(\theta_{1,2}(q)-\eta(q)^{3}\right), \quad \chi_{1}(q)=\frac{1}{2 \eta(q)}\left(\theta_{1,2}(q)+\eta(q)^{3}\right) .
$$

The problem is that these two characters do not have a good homogeneous transformation behavior under the modular group. More specifically, the transformation $S: \tau \rightarrow-1 / \tau$ will map these characters into functions, where $\tau$ appears directly as a prefactor, and not only via $q=\exp (2 \pi \mathrm{i} \tau)$. In other words, the $S$-matrix has entries which are not all constant with respect to $\tau$. We already know, however, that the vacuum representation is an irreducible sub-representation of a larger, indecomposable, representation $\mathcal{R}$. The point is that the character of the full indecomposable representation is well behaved,

$$
\chi_{\mathcal{R}}(q)=\frac{\theta_{1,2}(q)}{\eta(q)} .
$$

The modular transforms of the vacuum-character, however, can be given in terms of an $S$-matrix with constant coefficients, if we enlarge the set of characters by functions of the form

$$
\tilde{\chi}_{0}(q)=\frac{\log (q)}{2 \pi \mathrm{i}} \eta(q)^{2},
$$

where we choose form $\log (q)$ the branch that coincides with $2 \pi \mathrm{i} \tau$. This function cannot be interpreted as character, but it still is a valid torus amplitude. This is a manifestation of a more general fact, namely that the space of torus amplitudes and the space of characters are, as vector spaces, not any longer isomorphic, if indecomposable representations have to be taken into account, as is the case in LCFT.

\subsection{Periods and Torus Amplitudes}

We now come to the point where we can compare our results for the periods on the torus, obtained by a computation on a ramified covering of the sphere, with the well-known results in terms of elliptic functions, depending on the modulus $\tau$ of the torus. We thus will make some use of the theory of elliptic functions in order to compute torus amplitudes directly on the complex plane. Actually, we will do the computation on the two-sheeted covering of $\mathbb{C P}^{1}$ with four branch points $e_{1}, \ldots, e_{4}$. We will then translate the result to expressions in the modular parameter $\tau$ with the help of some identities for elliptic functions.

We recall that a torus is an elliptic curve defined by an equation

$$
y^{2}=\left(z-e_{1}\right)\left(z-e_{2}\right)\left(z-e_{3}\right)\left(z-e_{4}\right),
$$

where infinity is not taken as a branch point. We note that the branch points are related to the modular parameter $\tau$ in a non-trivial manner. Due to conformal invariance, we can fix three of the four branch points to arbitrarily chosen coordinates. However, we will not directly make use of this yet, but instead assume only that $e_{4}=\infty$ and $e_{2}+e_{3}+e_{1}=0$. It is easy to see that this is possible without loss of generality. Then, elementary symmetric polynomials in the $e_{i}$ can be expressed in terms of modular functions $g_{2}(\tau)$ and $g_{3}(\tau)$, which essentially are the Eisenstein series $E_{4}(\tau)$ and $E_{6}(\tau)$, respectively. This holds more generally, but is particularly easy in this setting, 
where we then have $y^{2}=4 z^{3}-g_{2} z-g_{3}$. Moreover, the so-called discriminant of the elliptic curve, which reads

$$
\Delta=\prod_{i<j}\left(e_{i}-e_{j}\right)^{2}
$$

up to an irrelevant numerical constant, is nothing else than the modular invariant $\Delta(\tau)=\eta(\tau)^{24}$. Here, $\eta(\tau)$ is the Dedekind $\eta$-function defined above.

The important point is that there is no simple relation between the branch points and the modular parameter $\tau$. However, one can make use of a so-called uniformizing variable $u$ which allows to define the elliptic curve in terms of an elliptic function, namely the Weierstrass' function $\wp$. One has

$$
\wp^{\prime}(u)=4 \wp^{3}(u)-g_{2} \wp(u)-g_{3} .
$$

This relation as well as some relations involving standard $\vartheta$-functions will be helpful later on.

We first will do an indirect computation: On the branched covering of $\mathbb{C P}^{1}$, the periods of the corresponding torus can be computed by contour integrations in the following way: Firstly, we choose a basis of homology cycles, where $\alpha$ encircles $e_{2}$ and $e_{3}$, and $\beta$ encircles $e_{1}$ and $e_{3}$. (This choice is conventional, and assumes that the branch cuts run between $e_{2}$ and $e_{3}$, and between $e_{1}$ and $e_{4}$, respectively.) Therefore,

$$
\begin{aligned}
& \pi_{\alpha}=\oint_{\alpha} \frac{\mathrm{d} z}{y}=\frac{\sqrt{2}}{2 \pi} \int_{e_{2}}^{e_{3}} \frac{\mathrm{d} z}{y}=\frac{\sqrt{2}}{2 \pi} \int_{e_{2}}^{e_{3}} \mathrm{~d} z \prod_{i=1}^{4}\left(e_{i}-z\right)^{-1 / 2} \\
& \pi_{\beta}=\oint_{\beta} \frac{\mathrm{d} z}{y}=\frac{\sqrt{2}}{2 \pi} \int_{e_{1}}^{e_{3}} \frac{\mathrm{d} z}{y}=\frac{\sqrt{2}}{2 \pi} \int_{e_{1}}^{e_{3}} \mathrm{~d} z \prod_{i=1}^{4}\left(e_{i}-z\right)^{-1 / 2}
\end{aligned}
$$

The correct proportionality factors connecting the contour integrations to line integrations follow from the theory of hypergeometric integrals. The above integrals look very much like Feigin-Fuks screening integrals in a free field representation of a CFT. Indeed, they are just that for the CFT of 1-differentials, the ghost systems with $c=-2$ which we use throughout this paper. More precisely, we have, with $\mu$ the $\mathbb{Z}_{2}$ twist field of conformal weight $h=-1 / 8$ simulating a branch point, that

$$
\frac{\sqrt{2}}{2 \pi} \int_{e_{k}}^{e_{l}} \mathrm{~d} z \prod_{i=1}^{4}\left(e_{i}-z\right)^{-1 / 2}=\prod_{i<j}\left(e_{i}-e_{j}\right)^{-1 / 4}\left\langle\mu\left(e_{1}\right) \mu\left(e_{2}\right) \mu\left(e_{3}\right) \mu\left(e_{4}\right)\right\rangle_{C_{\left(e_{k}, e_{l}\right)}},
$$

where we have to divide the correlator by its free part such that only the screening integration remains. It is clear that different line integrations lead to different linear combinations of conformal blocks, indicated here by the notation $C_{\left(e_{k}, e_{l}\right)}$ for a contour encircling only the branch points $e_{k}$ and $e_{l}$. Thus, we can write

$$
\begin{aligned}
& \pi_{\alpha}=\Delta^{-1 / 8}\left\langle\mu\left(e_{1}\right) \mu\left(e_{2}\right) \mu\left(e_{3}\right) \mu\left(e_{4}\right)\right\rangle_{(\alpha)}, \\
& \pi_{\beta}=\Delta^{-1 / 8}\left\langle\mu\left(e_{1}\right) \mu\left(e_{2}\right) \mu\left(e_{3}\right) \mu\left(e_{4}\right)\right\rangle_{(\beta)} .
\end{aligned}
$$

Now, with our choice of the homology basis, the periods of the torus should simply be $\pi_{\alpha} \sim 1$ and $\pi_{\beta} \sim \tau$. There is one subtlety to be taken care of, namely that we have computed the periods not in a flat metric, but in a singular metric of the branched covering of the complex plane. The periods depend on the metric due to the conformal anomaly. The difference between these two metrics is well known and amounts to $\prod_{i<j}\left(e_{i}-e_{j}\right)^{-1 / 12}=\Delta^{-1 / 24}=\eta^{-1}$. In the literature, this is often called the Liouville factor. Putting all together, we find the result

$$
\begin{aligned}
\Delta^{-1 / 8} \Delta^{1 / 24} \pi_{\alpha} & =\eta^{2} \cdot 1=\left\langle\mu\left(e_{1}\right) \mu\left(e_{2}\right) \mu\left(e_{3}\right) \mu\left(e_{4}\right)\right\rangle_{(\alpha)}, \\
\Delta^{-1 / 8} \Delta^{1 / 24} \pi_{\beta} & =\eta^{2} \cdot \tau=\left\langle\mu\left(e_{1}\right) \mu\left(e_{2}\right) \mu\left(e_{3}\right) \mu\left(e_{4}\right)\right\rangle_{(\beta)} .
\end{aligned}
$$

Therefore, we claim that the conformal blocks $\langle 1\rangle_{\gamma}$ on the torus are given by $\eta^{2}(\tau)$ for $\gamma=\alpha$, and by $\eta^{2}(\tau) \tau$ for $\gamma=\beta$. 
Now we rederive this result by a more direct computation: We could continue and evaluate the correlators. It is well known that these turn out to be proportional to the hypergeometric system ${ }_{2} F_{1}\left(\frac{1}{2}, \frac{1}{2} ; 1 ; x\right)$ with $x$ the anharmonic ratio of the four branch points. In principle, it is possible to express the resulting function in $x$ in terms of $\tau$ using the relation $x=\kappa^{2}(\tau)=\left(\vartheta_{2}(0 \mid \tau) / \vartheta_{3}(0 \mid \tau)\right)^{4}$, where $\vartheta_{i}(v \mid \tau), i=1 \ldots 4$, denote the standard Jacobi $\vartheta$-functions. These are given as special cases of the Hermite $\Theta$-function

$$
\Theta_{\mu, \nu}(v \mid \tau)=\sum_{n \in \mathbb{Z}} \exp \left[2 \pi \mathrm{i} \tau \frac{1}{2}\left(n+\frac{1}{2} \mu\right)^{2}+2 \pi \mathrm{i} v\left(n+\frac{1}{2} \mu\right)+\pi \mathrm{i} n \nu\right],
$$

namely $\vartheta_{1}(v \mid \tau)=\mathrm{i} \Theta_{-1,1}(v \mid \tau), \vartheta_{2}(v \mid \tau)=\Theta_{-1,0}(v \mid \tau), \vartheta_{3}(v \mid \tau)=\Theta_{0,0}(v \mid \tau)$, and $\vartheta_{4}(v \mid \tau)=\Theta_{0,1}(v \mid \tau)$. The inverse relation is

$$
\tau=\mathrm{i} \frac{{ }_{2} F_{1}\left(\frac{1}{2}, \frac{1}{2} ; 1 ; 1-x\right)}{{ }_{2} F_{1}\left(\frac{1}{2}, \frac{1}{2} ; 1 ; x\right)} .
$$

Relations between expressions in the modular parameter and expressions in terms of branch points are often called (generalized) Thomae's formulæ. We can make this more manifest by remembering that the complete elliptic integral of the first kind can be expressed in terms of a hypergeometric system, namely

$$
K(x)={ }_{2} F_{1}\left(\frac{1}{2}, \frac{1}{2} ; 1 ; x\right), \quad K^{\prime}(x)={ }_{2} F_{1}\left(\frac{1}{2}, \frac{1}{2} ; 1 ; 1-x\right) .
$$

On the other hand, one knows from the theory of elliptic curves that

$$
\begin{aligned}
K(x) & =\left(e_{1}-e_{3}\right)^{\frac{1}{2}} \omega & = & \frac{\pi}{2} \vartheta_{3}^{2}(0 \mid \tau), \\
K^{\prime}(x) & =-\mathrm{i}\left(e_{1}-e_{3}\right)^{\frac{1}{2}} \omega^{\prime} & = & -\mathrm{i} \frac{\pi}{2} \frac{\omega^{\prime}}{\omega} \vartheta_{3}^{2}(0 \mid \tau),
\end{aligned}
$$

where $\omega$ and $\omega^{\prime}$ are the two periods of the elliptic curve. Now, the four-point function can be explicitly evaluated up to an overall constant as

$$
\left\langle\mu(\infty) \mu\left(e_{1}\right) \mu\left(e_{2}\right) \mu\left(e_{3}\right)\right\rangle=-\pi\left(\frac{\left(e_{1}-e_{2}\right)\left(e_{2}-e_{3}\right)}{\left(e_{1}-e_{3}\right)}\right)^{\frac{1}{4}} F(x),
$$

where $F(x)$ is a linear combination in $K(x)$ and $K^{\prime}(x)$. Luckily, differences of branch points can be expressed in terms of $\vartheta$-functions as well. In addition to [83), we need

$$
\left(e_{2}-e_{3}\right)^{\frac{1}{2}}=\frac{\pi}{2 \omega} \vartheta_{2}^{2}(0 \mid \tau), \quad\left(e_{1}-e_{2}\right)^{\frac{1}{2}}=\frac{\pi}{2 \omega} \vartheta_{4}^{2}(0 \mid \tau) .
$$

Thus, the prefactor results in

$$
\left(\frac{\left(e_{1}-e_{2}\right)\left(e_{2}-e_{3}\right)}{\left(e_{1}-e_{3}\right)}\right)^{\frac{1}{4}}=\sqrt{\frac{\pi}{2 \omega}} \frac{\vartheta_{2} \vartheta_{4}}{\vartheta_{3}}=\sqrt{\frac{\pi}{2 \omega}} \frac{\vartheta_{2} \vartheta_{3} \vartheta_{4}}{\vartheta_{3}^{2}},
$$

where we abbreviate $\vartheta_{\alpha}=\vartheta_{\alpha}(0 \mid \tau)$. Making use of the relation

$$
\Delta^{\frac{1}{4}}=\frac{\pi^{3}}{4 \omega^{3}}\left(\vartheta_{2} \vartheta_{3} \vartheta_{4}\right)^{2}
$$

and plugging in the above formula for $K(x)$ and $K^{\prime}(x)$, we arrive at the statement

$$
\left\langle\mu(\infty) \mu\left(e_{1}\right) \mu\left(e_{2}\right) \mu\left(e_{3}\right)\right\rangle=-\pi \frac{\sqrt{2}}{\pi} \eta^{3}\left\{\begin{array}{r}
\omega, \\
(-\mathrm{i}) \omega^{\prime}
\end{array}\right.
$$

This is, up to the above mentioned Liouville factor $\prod_{i<j}\left(e_{i}-e_{j}\right)^{c / 12}$ and an overall scale equivalent to our first computation. As a further example we give without computation the result

$$
\left\langle\mu(\infty) \mu\left(e_{1}\right) \mu\left(e_{2}\right) \mu\left(e_{3}\right) V_{1}(z)\right\rangle=-\frac{1}{\eta^{6}} \sqrt{\frac{\pi^{3}}{\omega^{3}}} \frac{\vartheta_{1}^{4}(v \mid \tau)}{\vartheta_{1}(2 v \mid \tau)},
$$

where we have not yet divided out the Liouville factor, and where $v$ is related to $z$ via $z=\wp(v)$. The field $V_{1}(z)$ denotes here a vertex operator of charge $q=1$, such that its conformal weight is $h(q)=\frac{1}{2}\left(q^{2}+q\right)=1$. Since the total charge balance is already satisfied, no screening charge integrations are necessary. 


\subsection{Plane correlators and characters}

Finally, we will make some more direct connections of all these quantities and with the character functions for the $c=-2$ rational LCFT. The key to this is the relation (81) with its inverse, the elliptic modular function $x(\tau)=\kappa^{2}(\tau)$. If one wishes, $\kappa^{2}(\tau)$ can be expressed in terms of the Dedekind eta-function as

$$
\kappa^{2}(\tau)=16 \frac{\eta(2 \tau)^{16} \eta(\tau / 2)^{8}}{\eta(\tau)^{24}} .
$$

Obviously, the relation (81) is equivalent to the quotient of our two conformal blocks of four twist fields,

$$
\begin{aligned}
\tau & =\frac{\omega}{\omega^{\prime}}=\mathrm{i} \frac{\langle\mu(\infty) \mu(1) \mu(x) \mu(0)\rangle_{\beta}}{\langle\mu(\infty) \mu(1) \mu(x) \mu(0)\rangle_{\alpha}} \\
& =\mathrm{i}\left(\log (x)-4 \log (2)-\frac{1}{2} x-\frac{13}{64} x^{2}-\frac{23}{192} x^{3}-\frac{2701}{32768} x^{4}-\frac{5067}{81920} x^{5}-\ldots\right) .
\end{aligned}
$$

In fact, one finds the remarkable results that not only the quotient of the two conformal blocks can be expressed in modular quantities, but also the individual hypergeometric functions and the two conformal blocks themselves. First of all, one finds that

$$
{ }_{2} F_{1}\left(\frac{1}{2}, \frac{1}{2}, 1 ; \kappa^{2}\right)=\left(\vartheta_{3}\right)^{2}, \quad{ }_{2} F_{1}\left(\frac{1}{2}, \frac{1}{2}, 1 ; 1-\kappa^{2}\right)=-\mathrm{i} \tau\left(\vartheta_{3}\right)^{2},
$$

as functions of $\tau$. The prefactor of the two conformal blocks, however, does not have a simple expression in terms of $\vartheta_{\alpha}$ functions. It is now useful, to switch to the characters of the $c=-2$ theory. One has to note one subtlety here. Elliptic functions as the ones introduced above, are typically defined in the half-period $\tau$ with Fourier-expansions around $\tau=+\mathrm{i} \infty$ in the variable $\tilde{q}=\exp (\pi \mathrm{i} \tau)=q^{1 / 2}$, although this variable is denoted often with $q$ in the literature, causing considerable confusion. To start with, we have explicitly

$$
\begin{aligned}
& \vartheta_{3}=1+2 \sum_{n=1}^{\infty} \tilde{q}^{n^{2}}=\theta_{0,2}+\theta_{2,2}, \\
& \vartheta_{2}=2 \sum_{n=0}^{\infty} \tilde{q}^{(n+1 / 2)^{2}}=\theta_{1,2}
\end{aligned}
$$

as functions of $\tau$. It is worth noting that the characters of the irreducible representations for the twist fields appear only in their sum. The difference of these characters is also a meaningful quantity, namely

$$
\theta_{0,2}-\theta_{2,2}=\frac{\eta^{2}(\tau / 2)}{\eta(\tau)}=\vartheta_{4} \quad \text { or } \quad \chi_{-1 / 8}-\chi_{3 / 8}=\left(\frac{\eta(\tau / 2)}{\eta(\tau)}\right)^{2}
$$

With this in mind, we find the relations

$$
\begin{aligned}
{ }_{2} F_{1}\left(\frac{1}{2}, \frac{1}{2}, 1 ; \kappa^{2}\right) & =\left(\theta_{0,2}+\theta_{2,2}\right)^{2}=\left(\eta\left(\chi_{-1 / 8}+\chi_{3 / 8}\right)\right)^{2} \\
\left(\kappa^{2}\left(1-\kappa^{2}\right)\right)^{1 / 4} & =2 \frac{\theta_{1,2}\left(\theta_{0,2}-\theta_{2,2}\right)}{\left(\theta_{0,2}+\theta_{2,2}\right)^{2}}=2 \chi_{\mathcal{R}} \frac{\chi_{-1 / 8}-\chi_{3 / 8}}{\left(\chi_{-1 / 8}+\chi_{3 / 8}\right)^{2}}
\end{aligned}
$$

The latter is the prefactor of the conformal blocks. But it is also a correlation function in its own right, namely the function

$$
\begin{aligned}
& \left\langle\sigma(\infty) \mu(1) \mu\left(\kappa^{2}\right) \mu(0)\right\rangle=\left[\kappa^{2}\left(1-\kappa^{2}\right)\right]^{1 / 4} \\
& \quad=2 \tilde{q}^{1 / 4}\left(1-6 \tilde{q}+21 \tilde{q}^{2}-62 \tilde{q}^{3}-162 \tilde{q}^{4}-384 \tilde{q}^{5}+855 \tilde{q}^{6}+\ldots\right)
\end{aligned}
$$


where $\sigma(z)=\Phi_{-1 / 2}(z)$. Note that the charge balance is automatically fulfilled for this correlator, so no screening current integration is necessary. Collecting everything leads to the surprising compact result

$$
\begin{aligned}
\langle\mu \mu \mu \mu\rangle_{\alpha} & =2 \chi_{\mathcal{R}}(\tau) \eta^{2}(\tau / 2), \\
\langle\mu \mu \mu \mu\rangle_{\beta} & =2 \chi_{\mathcal{R}}(\tau) \tilde{\chi}_{0}(\tau / 2) .
\end{aligned}
$$

Up to a factor involving the Dedekind eta-function taken at the half-period, the conformal blocks turns out to be directly related to the character of the $h=0$ sector of the theory! We might have expected something like this, since the four-point function on the sphere should correspond to a zero-point function on the torus. Note that $\eta^{2}(\tau / 2)=\chi_{0}(\tau / 2)-\chi_{1}(\tau / 2)$. We pay a small price for using the picture of a ramified covering of the sphere. The metric or Liouville factor actually enters with the half-period instead of the full period reflecting the fact that there is an additional $\mathbb{Z}_{2}$ symmetry. The realization of a torus via a ramified covering introduces a double-valuedness which is not present, when the torus is realized as $\mathbb{C} / \Lambda$ with $\Lambda$ a lattice spanned by $\mathbb{Z} \omega \oplus \mathbb{Z} \omega^{\prime}$. The period or modulus $\tau$ can be expressed solely in terms of the characters of the representations, which are part of the indecomposable $h=0$ representation $\mathcal{R}$, namely

$$
\tau=\frac{1}{\mathrm{i} \pi} \frac{\tilde{\chi}_{0}}{\chi_{\mathcal{R}}}=\frac{1}{\mathrm{i} \pi} \frac{\tilde{\chi}_{0}}{\chi_{0}-\chi_{1}} .
$$

We complete this brief discussion by presenting the other possible four-point correlators involving twist fields. Since we need at least on $\mu$ field, as the excited twist $\sigma$ is degenerate of level four, the remaining two cases are, up to permutations,

$$
\begin{aligned}
\langle\sigma(\infty) \sigma(1) \mu(x) \mu(0)\rangle & =x[x(1-x)]^{-3 / 4}{ }_{2} F_{1}\left(-\frac{1}{2}, \frac{1}{2}, 1 ; x\right), \\
\langle\sigma(\infty) \sigma(1) \mu(x) \sigma(0)\rangle & =[x(1-x)]^{-1 / 4} \\
& =\frac{1}{2} \frac{\left(\chi_{-1 / 8}+\chi_{3 / 8}\right)^{2}}{\chi_{\mathcal{R}}\left(\chi_{-1 / 8}-\chi_{3 / 8}\right)},
\end{aligned}
$$

where $\frac{\pi}{2} 2 F_{1}\left(-\frac{1}{2}, \frac{1}{2}, 1 ; \kappa^{2}\right)=E\left(\kappa^{2}\right)$ is the complete elliptic integral of the second kind. All these functions are related to the vacuum character. They differ by a metric factor which can be expressed in terms of the Dedekind eta-function. If we define the symbol $(n)=\eta(n \tau / 2)=\eta\left(q^{n / 2}\right)$, then we can write all the different twist field four-point functions as

$$
\begin{aligned}
\langle\mu \mu \mu \mu\rangle & =\chi_{\mathcal{R}}(1)^{2}, \\
\langle\sigma \mu \mu \mu\rangle & =\chi_{\mathcal{R}} \frac{(1)^{6}(4)^{4}}{(2)^{10}}, \\
\langle\sigma \sigma \mu \mu\rangle & =\chi_{\mathcal{R}} \frac{(2)^{4} E_{2}\left(q^{2}\right)}{(1)^{6}}, \\
\langle\sigma \sigma \sigma \mu\rangle & =\chi_{\mathcal{R}} \frac{(2)^{11}}{(1)^{4}(4)^{7}},
\end{aligned}
$$

where we only give the conformal blocks for the $\alpha$ homology cycle and where we have skipped some irrelevant numerical factors. The third case, involving two excited twists, leads to the complete elliptic integral of the second kind. Expressing it in terms of the Jacobi theta-functions leads to the given result, where $E_{2}$ is the second Eisenstein series, $E_{2}(q)=1-24 \sum_{k \in \mathbb{N}} \sigma_{1}(k) q^{2 k}$ with $\sigma_{1}(k)$ the sum of the divisors of $k$.

It is possible to generalize such connections between four-point amplitudes on the sphere and torus vacuum amplitudes. The tool for this is uniformization. In case that one field in the four-point functions is degenerate of level two, we know that the four-point amplitude has to satisfy a secondorder differential equation. Thus, the four-point amplitude will be related to a hypergeometric 
functions ${ }_{2} F_{1}(a, b, c ; x)$. More precisely, if $\Phi_{h_{0}}$ is degenerate of level two, we can bring any fourpoint function involving this field into the form

$$
\begin{aligned}
\left\langle\Phi_{h_{3}}(\infty) \Phi_{h_{2}}(1) \Phi_{h_{1}}(0) \Phi_{h_{0}}(z)\right\rangle & =z^{p+\mu_{01}}(1-z)^{q+\mu_{20}} F(z), \\
\mu_{i j} & =\left(h_{0}+h_{1}+h_{2}+h_{3}\right) / 3-h_{i}-h_{j}, \\
p & =\frac{1}{6}-\frac{2}{3} h_{0}-\mu_{01}-\frac{1}{6} \sqrt{r_{1}}, \\
q & =\frac{1}{6}-\frac{2}{3} h_{0}-\mu_{01}-\frac{1}{6} \sqrt{r_{2}}, \\
r_{i} & =1-8 h_{0}+16 h_{0}^{2}+48 h_{i} h_{0}+24 h_{i} .
\end{aligned}
$$

The remaining function $F(z)$ then is a solution of the hypergeometric system ${ }_{2} F_{1}(a, b ; c ; z)$ given by

$$
\begin{aligned}
0 & =\left(z(1-z) \partial_{z}^{2}+[c-(a+b+1) z] \partial_{z}-a b\right) F(z), \\
a & =\frac{1}{2}-\frac{1}{6} \sqrt{r_{1}}-\frac{1}{6} \sqrt{r_{2}}-\frac{1}{6} \sqrt{r_{3}}, \\
b & =\frac{1}{2}-\frac{1}{6} \sqrt{r_{1}}-\frac{1}{6} \sqrt{r_{2}}+\frac{1}{6} \sqrt{r_{3}}, \\
c & =1-\frac{1}{3} \sqrt{r_{1}} .
\end{aligned}
$$

The general solution is then a linear combination of the two linearly independent solutions or conformal blocks ${ }_{2} F_{1}(a, b ; c ; z)$ and $z^{1-c}{ }_{2} F_{1}(a-c+1, b-c+1 ; 2-c ; z)$. Which linear combination one has to take is determined by the requirement that the full four-point function involving holomorphic and anti-holomorphic dependencies must be single-valued to represent a physical observable quantity.

Uniformization of this case can now be done with the help of a beautiful formula by Wirtinger, namely

$$
\frac{1}{2} \frac{\Gamma(2 b) \Gamma(c-b)}{\pi^{2 b} \Gamma(c)}{ }_{2} F_{1}\left(a, b, c ; \kappa^{2}(\tau)\right)=\left[\vartheta_{3}(0 \mid \tau)\right]^{4 b} \int_{0}^{1 / 2} \Phi(u, \tau) \mathrm{d} u,
$$

where the line integral is valid for $\Re \mathfrak{e} c>\Re \mathfrak{e} b>0$, defining a one-valued function regular in the half-plane $\Im \mathfrak{m} \tau>0$. In all other cases, the line integral must be replaced by a contour integration. The function $\Phi(u, \tau)$ is entirely defined in terms of the Jacobi theta-functions,

$$
\Phi(u, \tau)=\left[\frac{\vartheta_{1}(u \mid \tau)}{\vartheta_{1}^{\prime}(0 \mid \tau)}\right]^{2 b-1}\left[\frac{\vartheta_{2}(u \mid \tau)}{\vartheta_{2}(0 \mid \tau)}\right]^{2(c-b)-1}\left[\frac{\vartheta_{3}(u \mid \tau)}{\vartheta_{3}(0 \mid \tau)}\right]^{1-2 a}\left[\frac{\vartheta_{4}(u \mid \tau)}{\vartheta_{4}(0 \mid \tau)}\right]^{1-2(c-a)},
$$

where $\vartheta_{1}^{\prime}(v \mid \tau)=\partial_{v} \vartheta_{1}(v \mid \tau)$.

As a small example, we look at the Ising model with $c=c_{4,3}=1 / 2$. The field of conformal weight $h=h_{2,1}=1 / 2$ is degenerate of weight two. Its four-point function is a rational function, namely

$$
\left\langle\Psi_{2,1}(\infty) \Psi_{2,1}(1) \Psi_{2,1}(x) \Psi_{2,1}(0)\right\rangle=[x(1-x)]^{-1}{ }_{2} F_{1}\left(-2,-\frac{1}{3},-\frac{2}{3} ; x\right)=\frac{1-x+x^{2}}{x^{2}(1-x)^{2}} .
$$

Expanding this for $x=\kappa^{2}(\tau)$ results in the series

$$
\begin{aligned}
& \left\langle\Psi_{2,1}(\infty) \Psi_{2,1}(1) \Psi_{2,1}\left(\kappa^{2}\right) \Psi_{2,1}(0)\right\rangle= \\
& \quad \frac{1}{16 \tilde{q}}\left(1+8 \tilde{q}+276 \tilde{q}^{2}+2048 \tilde{q}^{3}+11202 \tilde{q}^{4}+49152 \tilde{q}^{5}+184024 \tilde{q}^{6}+\ldots\right)
\end{aligned}
$$

in the variable $\tilde{q}=q^{1 / 2}$. This is a known series, namely the McKay-Thompson series of class $4 \mathrm{~A}$ for the Monster, which is the character of the extremal vertex operator algebra of rank 12 . We find it most astonishing that this function appears within the Ising model!

The Rising model admits three irreducible representations, with conformal weight $h_{1,1}=0$, $h_{2,1}=1 / 2$ and $h_{1,2}=1 / 16$, respectively. The characters are all given in terms of the modular 
functions

$$
\begin{aligned}
K_{p, p^{\prime}, r, s} & =\frac{1}{\eta(q)} \sum_{n \in \mathbb{Z}} q^{\left(2 p p^{\prime} n+p r-p^{\prime} s\right)^{2} / 4 p p^{\prime}}=\frac{\theta_{p r-p^{\prime} s, p p^{\prime}}(q)}{\eta(q)} \\
\chi_{p, p^{\prime}, r, s} & =K_{p, p^{\prime}, r, s}-K_{p, p^{\prime}, r,-s}=\frac{\theta_{p r-p^{\prime} s, p p^{\prime}}(q)-\theta_{p r+p^{\prime} s}(q)}{\eta(q)}
\end{aligned}
$$

with the functions defined in (66). These expressions yield the characters for all representations from the conformal grid $h_{r, s}$ of all minimal models $c_{p, p^{\prime}}$. Expressing these in $\tilde{q}$, one finds the remarkable identity

$$
\left\langle\Psi_{2,1}(\infty) \Psi_{2,1}(1) \Psi_{2,1}\left(\kappa^{2}\right) \Psi_{2,1}(0)\right\rangle=\left(\chi_{4,3,1,1}+\chi_{4,3,2,1}\right)^{24}-1
$$

up to an irrelevant numerical factor. But the most remarkable property of this four-point function is that it is closely related to the modular invariant $J(\tau)$, namely

$$
J(\tau)=\frac{4}{27} \frac{\left(1-\kappa^{2}+\kappa^{4}\right)^{3}}{\kappa^{4}\left(1-\kappa^{2}\right)^{2}}=\frac{4}{27}\left(1-\kappa^{2}+\kappa^{4}\right)^{2}\left\langle\Psi_{2,1}(\infty) \Psi_{2,1}(1) \Psi_{2,1}\left(\kappa^{2}\right) \Psi_{2,1}(0)\right\rangle .
$$

Of course, we do not get simple relations between the four-point functions of a given CFT and its torus vacuum-amplitudes, as we found in the case of the special theory with $c=-2$. The reason is that the branched covering of the Riemann sphere, generated by the fields in an arbitrary four-point functions, is not necessarily a torus. In fact, it almost never is. The reader should keep in mind that even in the case that (at least) one of the fields is degenerate of level two such that one screening integration has to be performed, does not imply that we have the geometry of a torus. On the contrary, except for $c=-2$, where the contours can indeed be chosen as homology cycles, we are almost always forced to use Pochhammer double loops to get a contour which closes. However, what the uniformization tells us is that we can view the geometries generated by the four fields in the correlator as a torus-like branched covering of some non-trivial Riemann surface. Let us make this a bit more precise by looking at another four-point function in the Ising model, namely at the correlator of the field $\Phi_{1,2}$ with $h=h_{1,2}=1 / 16$ which is also degenerate of level two. We find

$$
\left\langle\Phi_{1,2}(\infty) \Phi_{1,2}(1) \Phi_{1,2}(x) \Phi_{1,2}(0)\right\rangle=[x(1-x)]_{2}^{-1 / 8} F_{1}\left(-\frac{1}{4}, \frac{1}{4}, \frac{1}{2} ; x\right) .
$$

Making use of one of the elementary relations for hypergeometric functions, this can be expressed as

$$
\left\langle\Phi_{1,2}(\infty) \Phi_{1,2}(1) \Phi_{1,2}\left(\kappa^{2}\right) \Phi_{1,2}(0)\right\rangle=\frac{1}{\sqrt{2}}\left[\kappa^{2}\left(1-\kappa^{2}\right)\right]^{-1 / 8} \sqrt{1+\sqrt{1-\kappa^{2}}} .
$$

Comparing this with the expansions in the elliptic modulus $\kappa^{2}$ for the correlation functions of the $c=-2$ theory, we see that the spin four-point correlation function of the Ising model goes with $\left(\kappa^{2}\right)^{1 / 8}$, while all twist field correlators in the $c=-2$ model go with $\left(\kappa^{2}\right)^{1 / 4}$. So, there is an additional multi-valuedness, an additional branching.

\section{Conclusion}

We have collected here various issues concerning logarithmic conformal field theory. We concentrated on the best known example of a LCFT, namely the rational theory with $c=c_{2,1}=-2$. The reason for this is that we were mainly interested in the geometrical meaning of the logarithmic operators. This particular conformal field theory is different to other conformal field theories such as the minimal models, in the sense that its fields have an immediate geometrical meaning, such as branch points or poles, inserted in the complex plane. Thus, field insertion very directly change the geometry from the complex plane to a ramified covering of it. This works much more naturally than in other CFTs, where the ramified coverings are defined only locally and screening contours do not close, when one attempts to choose simple loops encircling two field insertions. Instead, in the generic CFT case, one has to take Pochhammer double loops. 
In the LCFT setting discussed here, screening contours can be chosen as ordinary homology cycles. But this implies that logarithmic operators must appear. Considering branch points as certain vertex operators, and then viewing these vertex operators as dynamical objects we can move around, leads to a possible degenerate case. When we let two branch points run into each other, a homology cycle might get pinched between them. If the two branch points have monodromies, which cancel each other, the pinched cycle leads to a defect, since it cannot be closed on the same sheet. Deforming the contour leaves one with a small line integral of a simple pole, giving rise to the logarithmic divergences one observes in LCFT.

After having interpreted the origin of logarithms in a geometrical way, we consider two settings where moving around of branch points plays an important role. Firstly, we look at the SeibergWitten solutions of supersymmetric low energy effective field theories. It is a well known fact that the periods of a natural meromorphic one-form, defined on a hyperelliptic surface, either vanish or exhibit a logarithmic behavior, when certain homology cycles shrink to zero size. This behavior is, of course and naturally, precisely recovered, if one computes the periods in terms of LCFT correlators of the $c=-2$ system of analytic one-differentials.

The second interesting topic we wished to raise was a direct comparison between quantities one can compute directly on the torus, and plane correlations functions with four $\mathbb{Z}_{2}$ twist fields inserted to simulate the torus. We have tried to make some of these connections very explicit. However, we believe that this view point is rather uncommon, and so, not much is yet known about such relations. In particular, almost nothing can be said for similar considerations on other CFTs. However, we are convinced that such connections between ramified coverings of the plane and nontrivial Riemann surfaces, in particular the torus, must generally exist. We leave the exploration of this issue to future work.

Last but not least, we hope that this small contribution, attempting to discuss various topics in physics and to make use of mathematical structures from many fields, combining some things in slightly uncommon ways, is a bit in the spirit of Ian Kogan, circumnavigating a very small part of physics.

\section{Acknowledgments}

Throughout the years, I have enjoyed many fruitful discussions with and comments from Nikolas Akerblom, Ralph Blumenhagen, Andreas Bredthauer, John Cardy, Holger Eberle, Matthias Gaberdiel, Amihay Hanani, Andreas Honecker, Marco Krohn, Neil Lambert, Alex Nichols, Werner Nahm, Klaus Osterloh, Hubert Saleur, Julia Voelskow, Gerard Watts, Peter West, John Wheater, and, of course, Ian Kogan.

\section{A Structure Constants}

structure constants of the $c_{p, 1}$ LCFTs. These are needed, if correlation functions are decomposed into 2- 3- and 4-point functions. The case important for this paper is $p=2$. One starts from an ansatz for the OPE,

$$
\Phi_{q}(z) \Phi_{q^{\prime}}(w)=\sum_{q^{\prime \prime}} C_{q, q^{\prime}}^{q^{\prime \prime}}(z-w)^{h\left(q^{\prime \prime}\right)-h(q)-h\left(q^{\prime}\right)} \Phi_{q^{\prime \prime}}(w)+\ldots
$$

where we omitted logarithmic contributions and descendants and where $q, q^{\prime}, q^{\prime \prime}$ all are admissible charges according to (17). The local primary fields decompose into chiral vertex operators with coefficients determined by the braid matrices of the BRST-invariant vertex operators. Depending on whether the local primary fields are chiral or not, we put $\chi=1,0$ respectively and have

$$
\left(\mathcal{D}_{\left(n, n^{\prime}\right),\left(m, m^{\prime}\right)}^{\left(l, l^{\prime}\right)}\right)^{1+\chi}=\frac{N_{\left(l, l^{\prime}\right)\left(l, l^{\prime}\right)}^{(1,1)}}{N_{\left(n, n^{\prime}\right)\left(n, n^{\prime}\right)}^{(1,1)} N_{\left(m, m^{\prime}\right)\left(m, m^{\prime}\right)}^{(1,1)}}\left(\Delta_{n, m}^{l}(x) \Delta_{n^{\prime}, m^{\prime}}^{l^{\prime}}\left(x^{\prime}\right)\right)^{1+\chi},
$$




$$
\begin{aligned}
\Delta_{n, m}^{l}(x) & =(-1)^{\frac{1}{2}(n+m-l-1)}\left(\frac{[n]_{x}[m]_{x}[l]_{x}}{[1]_{x}}\right)^{\frac{1}{2}} \\
& \times \prod_{j=(l+n-m+1) / 2}^{n-1}[j]_{x} \prod_{j=(m+n-l+1) / 2}^{n-1}[j]_{x} \prod_{j=(l+m-n+1) / 2}^{(l+m+n-1) / 2} \frac{1}{[j]_{x}}
\end{aligned}
$$

where the brackets are given by $[j]_{x}=x^{j / 2}-x^{-j / 2}$ with $x=\exp \left(\pi \mathrm{i} \alpha_{+}^{2}\right)$ and $x^{\prime}=\exp \left(\pi \mathrm{i} \alpha_{-}^{2}\right)$. In the case of interest, $\alpha_{+}^{2}=2 p$ and $\alpha_{-}^{2}=-2 / p$. These formulæ are valid if the normalization of the two-point functions simply is chosen to be

$$
\left\langle\Psi_{n, n^{\prime}}(z) \Psi_{m, m^{\prime}}(w)\right\rangle=\frac{\delta_{n, m} \delta_{n^{\prime}, m^{\prime}}}{(z-w)^{2 h_{n, n^{\prime}}}},
$$

where we denote primary fields from the Kac-table by $\Psi$ instead of $\Phi$. The general normalization constants $N_{\left(n, n^{\prime}\right)\left(m, m^{\prime}\right)}^{\left(l, l^{\prime}\right)}=\left\langle h_{l, l^{\prime}}\left|V_{\left(n, n^{\prime}\right)\left(m, m^{\prime}\right)}^{\left(l, l^{\prime}\right.}(1)\right| h_{m, m^{\prime}}\right\rangle$ can be expressed in terms of hypergeometric integrals (the contour integration of the screening currents around chiral vertex operators of a free field theory) and are given here for completeness:

$$
\begin{aligned}
& N_{\left(n, n^{\prime}\right)\left(m, m^{\prime}\right)}^{\left(l, l^{\prime}\right)}=(-1)^{\frac{1}{2}\left(\left(2 n^{\prime}-1\right) r+(2 n-1) r^{\prime}\right)} \alpha_{+}^{4 r r^{\prime}} 2^{-2 r r^{\prime}} \\
& \quad \times \prod_{j^{\prime}=1}^{r^{\prime}} \frac{\left[m^{\prime}-j^{\prime}\right]_{x^{\prime}}\left[j^{\prime}\right]_{x^{\prime}}}{[1]_{x^{\prime}}} \prod_{j=1}^{r} \frac{[m-j]_{x}[j]_{x}}{[1]_{x}} \\
& \times \prod_{j^{\prime}=1}^{r^{\prime}} \frac{\Gamma\left(j^{\prime} \alpha_{-}^{2} / 2\right) \Gamma\left(m+\left(j^{\prime}-m^{\prime}\right) \alpha_{-}^{2} / 2\right) \Gamma\left(n+\left(j^{\prime}-n^{\prime}\right) \alpha_{-}^{2} / 2\right)}{\Gamma\left(\alpha_{-}^{2} / 2\right) \Gamma\left(m+n-2 r+\left(r^{\prime}-m^{\prime}-n^{\prime}+j^{\prime}\right) \alpha_{-}^{2} / 2\right)} \\
& \times \prod_{j=1}^{r} \frac{\Gamma\left(j \alpha_{+}^{2} / 2-r^{\prime}\right) \Gamma\left(m^{\prime}-r^{\prime}+(j-m) \alpha_{+}^{2} / 2\right) \Gamma\left(n^{\prime}-r^{\prime}+(j-n) \alpha_{+}^{2} / 2\right)}{\Gamma\left(\alpha_{+}^{2} / 2\right) \Gamma\left(m^{\prime}-r^{\prime}+n^{\prime}+(r-m-n+j) \alpha_{+}^{2} / 2\right)}
\end{aligned}
$$

where $l=n+m-2 r-1$ and similar for $l^{\prime}$. The structure constants of the OPE or equivalently of the Lie-algebra of the Fourier modes of the chiral fields are then given by

$$
C_{\left(n, n^{\prime}\right)\left(m, m^{\prime}\right)}^{\left(l, l^{\prime}\right)}=\mathcal{D}_{\left(n, n^{\prime}\right)\left(m, m^{\prime}\right)}^{\left(l, l^{\prime}\right)} N_{\left(n, n^{\prime}\right)\left(m, m^{\prime}\right)}^{\left(l, l^{\prime}\right)}
$$

These expressions have to be evaluated carefully, due to zeroes in numerator and denominators which arise when $k \alpha_{ \pm}^{2} / 2 \in \mathbb{Z}$ for $k \in \mathbb{Z}$. However, it turns out that all these zeroes cancel nicely, leaving us with well-defined and non-singular structure constants, when they are considered as the limit $C_{\left(n, n^{\prime}\right)\left(m, m^{\prime}\right)}^{\left(l, l^{\prime}\right)}(p)=\lim _{\varepsilon \rightarrow 0} C_{\left(n, n^{\prime}\right)\left(m, m^{\prime}\right)}^{\left(l, l^{\prime}\right)}(p+\varepsilon)$ for $\alpha_{+}^{2} / 2=p \in \mathbb{Z}$. For example, we have for the chiral local primary fields

$$
\begin{aligned}
& \left(C_{(n, 1)(m, 1)}^{(l, 1)}\right)^{2}=\left(\begin{array}{c}
\frac{1}{2}(n+m-|n-m|-2) \\
\frac{1}{2}(l-|n-m|-1)
\end{array}\right)^{-1} \varphi\left(\Delta_{n, m}^{l}(x)\right)^{2} \\
& \quad \times \quad \prod_{j=1}^{(n+m-l-1) / 2} \frac{(p j-1) !^{2}\left(p\left(\frac{1}{2}(n+m-l-1)+l+1-j\right)-2\right) !^{2}}{(p(m-j)-1) !^{2}(p(n-j)-1) !^{2}} \\
& \quad \times \prod_{j=1}^{n-1} \frac{(p(n-j)-1) !^{2}}{(p j-1) !(p(n-j+1)-2) !} \prod_{j=1}^{m-1} \frac{(p(m-j)-1) !^{2}}{(p j-1) !(p(m-j+1)-2) !} \\
& \quad \times \prod_{j=1}^{l-1} \frac{(p j-1) !(p(l-j+1)-2) !}{(p(l-j)-1) !^{2}}
\end{aligned}
$$


where $\varphi\left(\Delta_{n, m}^{l}\right)$ denotes the phase part of $\Delta_{n, m}^{l}$, which in this case is just a sign:

$$
\begin{gathered}
\Delta_{n, m}^{l}(x)=(-1)^{l p}(-1)^{\frac{1}{2}(n+m-l-1)(p+1)}(-1)^{p\left((l m+l n+n m) / 2-\left(l^{2}+m^{2}+n^{2}-1\right) / 4\right)} \\
\times \frac{\sqrt{n m l}(n-1) ! 2\left(\frac{1}{2}(l+m-n-1)\right) !}{\left(\frac{1}{2}(l+n-m-1)\right) !\left(\frac{1}{2}(m+n-l-1)\right) !\left(\frac{1}{2}(l+m+n-1)\right) !} .
\end{gathered}
$$

The other structure constants are not so easily expressed in a closed form without transcendent functions. Also, some multiplicities have to be taken into account, which stem from the $S U(2)$ structure of the extended chiral symmetry algebra. For instance, our fields $\sigma$ and $J$ of the $c=-2$ LCFT are actually spin doublets with respect to this $S U(2)$ structure. We present here the complete set of non-trivial OPEs for $c=-2$, i.e. $p=2$, in a graphical form in figure 2 (omitting the canonical dependencies on the coordinates) which directly shows the geometrical meaning of them. Thus, one can easily read off from these graphs that, e.g., the OPE of two twist fields $\mu$ either yields the identity $\mathbb{I}$ or the puncture operator $P$, depending on whether the two twist fields belong to two different branch cuts or are joined by a common branch cut.

As is generally the case in LCFTs, highest weight representations are no longer necessarily irreducible, but may possess a non-trivial Jordan-cell structure. In the case of the $c_{p, 1}$ models, where the Jordan-cells are of rank two, this results in the following general form of the OPE:

$$
\begin{aligned}
& \Psi_{r, s}(z) \Psi_{r^{\prime}, s^{\prime}}(w) \sim \sum_{\substack{R=\left|r-r^{\prime}\right|+1 \\
r+r^{\prime}-1 \equiv R \bmod 2}}^{r+r^{\prime}-1} \sum_{\substack{S=\left|s-s^{\prime}\right|+1 \\
s+s^{\prime}-1 \equiv S \bmod 2}}^{s+s^{\prime}-1} C_{(r, s),\left(r^{\prime}, s^{\prime}\right)}^{(R, S)}(z-w)^{-h_{r, s}-h_{r^{\prime}, s^{\prime}}} \\
& \times \sum_{|\{k\}| \geq-1-\left(\Delta h_{R, S}\right)} a_{(r, s),\left(r^{\prime}, s^{\prime}\right)}^{(R, S) ;\{k\}}\left(\partial^{-1-\left(\Delta h_{R, S}\right)-|\{k\}|}(z-w)^{h_{R, S}^{\min }-1}\right) \\
& \times\left(\mathcal{L}_{-\{k\}} \Psi_{R, S}(w)\right),
\end{aligned}
$$

including only "descendants" with respect to the stress energy tensor and ignoring "descendants" with respect to other chiral local fields such as $J$ and $W$. Here, $\mathcal{L}_{-\{k\}} \Psi_{R, S}=L_{-k_{1}} L_{-k_{2}} \ldots L_{-k_{n}}$ is a level $|\{k\}|=\sum_{i} k_{i}$ "descendant" of the primary field $\Psi_{R, S}$, whose conformal scaling dimension differs by an integer from the one of $\Psi_{R, S}$. They form Jordan blocks precisely when $q_{R, S} \in \mathbb{Z}_{+}$. We then have the following relation between the conformal scaling dimensions of the field $\Psi_{R, S}$ and the lowest primary field of the Jordan block: $h_{R, S}=h_{R, S}^{\min }+\left(\Delta h_{R, S}\right)$ with $\left(\Delta h_{R, S}\right) \in \mathbb{Z}_{+}$. For example, our field $P=\Psi_{1,3}$ has $q_{1,3}=1$ but $\left(\Delta h_{1,3}\right)=0$ such that $(z-w) \partial P=L_{0} P=\mathbb{I}$. Hence $P$ and II span the rank two Jordan cell indecomposable highest weight representation with the following action of the Virasoro zero mode $L_{0}$ on it:

$$
L_{0}|h\rangle=h|h\rangle, \quad L_{0}|\tilde{h}\rangle=h|\tilde{h}\rangle+|h\rangle,
$$

where in this case $h=0$, i.e. $P(z)|0\rangle=|\tilde{0}\rangle$. This can be seen explicitly from the OPE of two twist fields: As apparent from the above formula for the OPE,

$$
\begin{aligned}
\mu(z) \mu(w) & \sim(z-w)^{-\frac{1}{4}}\left[C_{\mu, \mu}^{\mathbb{I}} \mathbb{I}+C_{\mu, \mu}^{P}\left(P(w)+a_{\mu, \mu}^{P ;\{0\}}\left(\partial^{-1} \frac{1}{z-w}\right) L_{0} P(w)\right)\right]+\ldots \\
& \sim(z-w)^{-\frac{1}{4}}[\mathbb{I}+P(w)-2 \log (z-w) \mathbb{I}]+\ldots
\end{aligned}
$$

producing the desired logarithmic dependency which indicates the presence of a non-trivial Jordan cell structure for $P(w)$ and its "descendant", the identity field $L_{0} P(w)=\mathbb{I}$.

The coefficients $a_{(r, s),\left(r^{\prime}, s^{\prime}\right)}^{(R, S)}$ can be determined recursively, since they are entirely fixed by conformal invariance. If one rewrites the above OPE formula A.8 by expressing the $\mathcal{L}_{-\{k\}} \Phi$ as linear combinations of derivative terms $\partial^{\ell} \Phi$, one obtains in the case of chiral local fields ${ }^{1}$ the rather simple

\footnotetext{
${ }^{1}$ (quasi-) primary chiral fields are local iff their conformal scaling dimensions $h \in \frac{1}{2} \mathbb{Z}$.
} 
closed form of the corresponding coefficients

$$
\tilde{a}_{(r, s),\left(r^{\prime}, s^{\prime}\right)}^{(R, S) ; \ell}=\left(\begin{array}{c}
h_{r, s}+h_{r^{\prime}, s^{\prime}}-h_{R, S}-1+\ell \\
\ell
\end{array}\right)\left(\begin{array}{c}
2 h_{R, S}-1+\ell \\
\ell
\end{array}\right)^{-1}
$$

for $\ell \geq 0$. The general case can be obtained by analytic continuation, taking into account that the Laurent expansion of a non-local chiral field receives contributions with fractional powers. This does not pose a problem in our case, where the fields of the CFT naturally live on a Riemann surface, i.e. on an $p$-sheeted covering of the complex plane allowing for generalized Laurent expansions with fractional powers $\alpha \in \frac{1}{p} \mathbb{Z}_{p}$. This is the reason for the "offset" $\partial^{-1-\left(\Delta h_{R, S}\right)}(z-w)^{h_{R, S}^{\min }-1}$ in (A.8) instead of simply $(z-w)^{0}=1$, on which $\partial^{-|\{k\}|}$ acts. Similar corrections apply to the generalization of A.11.

Moreover, even when $(\Delta h) \notin \mathbb{Z}$, fields might still be linked by Jordan-cell structures with respect to other quantum numbers. In fact, the $c_{p, 1}$ LCFTs have the charge $q$ as a further such quantum number with respect to the current $J$. As one can easily infer from the form (26) of the OPE valid for reduced correlation functions, fields whose charges differ by integers may also be linked. And indeed, we have in the $c=-2$ case that the excited twist fields $\sigma, \tau$ are both linked to $\mu$. This is the reason, why we had to include $\mu$ and $\sigma$ into the right hand side of the OPE $\mu: J^{2}(w):=\mu(z) W(w)$ as generalized "descendants" of the field $\tau$. The generalization of (A.8) for enlarged chiral symmetry algebras is straight forward though cumbersome, and is therefore omitted.

The geometrical meaning of the logarithmic divergencies and the Jordan cell structure in the hyperelliptic case (i.e. $p=2$ ) is that the branched covering picture of a Riemann surface, which intrinsically is not smooth, is ambiguous in the case of two branch points flowing together. Besides yielding the equivalent of a puncture, this situation also corresponds to an asymptotic region in the moduli space of Riemann surfaces, where one handle becomes pinched. This latter possibility is accounted for by the logarithmic term in the OPE of two branch point fields. We have sketched the three different situations of fusing two branch points in figure 1. The other possible configurations of the basic fields of the $c=-2$ logarithmic CFT and their OPEs (including logarithmic terms) can be inferred in a similar fashion.

When OPEs are regularized by discarding their singular parts (normal ordering), the logarithmic divergencies will be lost. In the hyperelliptic case, normal ordering of two twist fields $\mu$ at a common branch cut replaces the two confluent branch points by a simple pole. Hence, normal ordering can be used to degenerate the moduli space of, say, $\left(N_{c}, N_{f}\right)$ supersymmetric Yang-Mills theories to the one of $\left(N_{c}-1, N_{f}+1\right)$ theories, thereby creating a matter hypermultiplet. The created hypermultiplets might even be massive, if we relax the condition that the sum over all branch points must vanish with the help of a global translation. Translation is a global conformal transformation and hence satisfies a Ward identity. However, due to logarithmic divergencies in LCFTs, the conformal Ward identity becomes modified, $L_{0}\langle\ldots\rangle=k\langle\ldots\rangle$ with $k$ a non-zero constant. But accompanying a global translation of all branch points such that a subset of them sums to zero by appropriate normal ordering of the complement subset cancels $k$ and creates massive hypermultiplets instead.

\section{B Multiple Hypergeometric Functions}

In this Appendix we collect some useful results on the generalized hypergeometric functions relevant for our purposes. This is mainly the so-called fourth Lauricella function $F_{D}$. To begin with, let $(a)_{n}$ be defined for $a \in \mathbb{C}, n \in \mathbb{Z}_{+}$as

$$
(a)_{n}=\prod_{k=0}^{n-1}(a+k)
$$

Of course, this is equivalent to $(a)_{n}=\Gamma(a+n) / \Gamma(a)$, which also provides an extension of the definition to arbitrary $n \in \mathbb{Z}$. Clearly, $(1)_{n}=n$ ! for $n \geq 0$. In 1893, Lauricella generalized hypergeometric functions to the case of many variables and defined in particular four multiple 
series, $F_{A}, \ldots, F_{D}$, which carry his name. The one important to us is $F_{D}$ which is defined as

$$
\begin{aligned}
& F_{D}^{(n)}\left(a, b_{1}, b_{2}, \ldots, b_{n}, c ; x_{1}, x_{2}, \ldots, x_{n}\right)= \\
& \quad \sum_{m_{1}=0}^{\infty} \sum_{m_{2}=0}^{\infty} \ldots \sum_{m_{n}=0}^{\infty} \frac{(a)_{m_{1}+m_{2}+\ldots+m_{n}}\left(b_{1}\right)_{m_{1}}\left(b_{2}\right)_{m_{2}} \ldots\left(b_{n}\right)_{m_{n}}}{(c)_{m_{1}+m_{2}+\ldots+m_{n}}(1)_{m_{1}}(1)_{m_{2}} \ldots(1)_{m_{n}}} x_{1}^{m_{1}} x_{2}^{m_{2}} \ldots x_{n}^{m_{n}}
\end{aligned}
$$

where, for convergence, we must have $\left|x_{1}\right|,\left|x_{2}\right|, \ldots,\left|x_{n}\right|<1$. For $n=1$, this function reduces to the ordinary Gauss hypergeometric function ${ }_{2} F_{1}\left(a, b_{1} ; c ; x_{1}\right)$, and for $n=2$, it is nothing else than the Appell function $F_{1}\left(a ; b_{1}, b_{2} ; c ; x_{1}, x_{2}\right)$.

Next, we will provide an integral representation of this Lauricella function which is of the Euler or Pochhammer type. The point is that it is only a single integral which means that the function $F_{D}$ is, in fact, the general solution of arbitrary $(n+3)$-point correlation functions with at least one field degenerate of level two. Let us consider the integral

$$
I=\int_{0}^{1} u^{a-1}(1-u)^{c-a-1} \prod_{i=1}^{n}\left(1-u x_{i}\right)^{-b_{i}} \mathrm{~d} u .
$$

Expanding via the generalized binomial theorem, we have

$$
\begin{aligned}
& I=\sum_{m_{1}=0}^{\infty} \ldots \sum_{m_{n}=0}^{\infty} \int_{0}^{1} u^{a-1}(1-u)^{c-a-1} \prod_{i=1}^{n} \frac{\left(b_{i}\right)_{m_{i}}}{(1)_{m_{i}}} u^{m_{i}} x^{m_{i}} \mathrm{~d} u \\
& =\sum_{m_{1}=0}^{\infty} \ldots \sum_{m_{n}=0}^{\infty} \frac{\left(b_{1}\right)_{m_{1}} \ldots\left(b_{n}\right)_{m_{n}}}{(1)_{m_{1}} \ldots(1)_{m_{n}}} x_{1}^{m_{1}} \ldots x_{n}^{m_{n}} \int_{0}^{1} u^{a+m_{1}+\ldots+m_{n}-1}(1-u)^{c-a-1} \mathrm{~d} u \\
& =\sum_{m_{1}=0}^{\infty} \ldots \sum_{m_{n}=0}^{\infty} \frac{\left(b_{1}\right)_{m_{1}} \ldots\left(b_{n}\right)_{m_{n}}}{(1)_{m_{1}} \ldots(1)_{m_{n}}} x_{1}^{m_{1}} \ldots x_{n}^{m_{n}} \frac{\Gamma\left(a+m_{1}+\ldots+m_{n}\right) \Gamma(c-a)}{\Gamma\left(c+m_{1}+\ldots+m_{n}\right)} \\
& =\frac{\Gamma(a) \Gamma(c-a)}{\Gamma(c)} F_{D}^{(n)}\left(a, b_{1}, \ldots, b_{n}, c ; x_{1}, \ldots, x_{n}\right) .
\end{aligned}
$$

Indeed, $F_{D}$ satisfies the following system of partial differential equations of second order, which can be recognized as the differential equations of a $(n+3)$-point correlation function containing one field degenerate of level two:

$$
\left[\left(1-x_{j}\right) \sum_{k=1}^{n} x_{k} \frac{\partial^{2}}{\partial x_{k} \partial x_{j}}+\left(c-\left(a+b_{j}+1\right) x_{j}\right) \frac{\partial}{\partial x_{j}}-b_{j} \sum_{\substack{k=1 \\ k \neq j}}^{n} x_{k} \frac{\partial}{\partial x_{k}}-a b_{j}\right] F=0
$$

where $F=F_{D}^{(n)}\left(a, b_{1}, \ldots, b_{n}, c ; x_{1}, \ldots, x_{n}\right)$ and $j=1, \ldots, n$. If hypermultiplets are present, i.e. $N_{f}>0$, then the corresponding $c=-2$ CFT correlation functions will contain $P\left(x_{j}\right)$ fields which, despite of possessing scaling dimension $h(P)=0$, are degenerate of level three. Consequently, the reduced $\quad$ correlators $\quad\left\langle\prod_{i=1}^{2 g+2} \mu\left(x_{i}\right) \prod_{j=1}^{r} J\left(x_{2 g+2+j}\right) \prod_{k=1}^{s} P\left(x_{2 g+2+r+k}\right)\right\rangle$ satisfy the following system of third order differential equations $(n=2 g+r+s-1)$ :

$$
\left[\frac{\partial^{2}}{\partial x_{j}^{2}}-\sum_{\substack{k=1 \\ k \neq j}}^{n+3}\left(\frac{2 h_{k}}{\left(x_{j}-x_{k}\right)^{2}}+\frac{2}{x_{j}-x_{k}} \frac{\partial}{\partial x_{k}}\right)\right] \frac{\partial}{\partial x_{j}} \prod_{1 \leq l<m \leq n+3}\left(x_{l}-x_{m}\right)^{q_{l} q_{m}}\langle\ldots\rangle=0
$$

with $j=2 g+2+r, \ldots, n+3$. Hence, we obtain in particular the following result for an arbitrary period of an Abelian form on a genus $g$ Riemann surface (the prefactors are determined via (25):

$$
\begin{aligned}
& \left\langle\left\langle\mu(\infty) \mu(0) \mu(1) \mu\left(x_{1}\right) \ldots \mu\left(x_{2 g-1}\right) J\left(z_{1}\right) \ldots J\left(z_{r}\right) P\left(p_{1}\right) \ldots P\left(p_{s}\right): P^{Q}(\varpi):\right\rangle\right. \\
& \quad=\pi \prod_{i=1}^{2 g-1}\left(x_{i}\right)^{-\frac{1}{2}} \prod_{j=1}^{r}\left(z_{j}\right)^{1} \prod_{k=1}^{s}\left(p_{k}\right)^{-1}(\varpi)^{g+s-r-1}
\end{aligned}
$$




$$
\begin{gathered}
\times F_{D}^{(n+1)}(\underbrace{\frac{1}{2}, \ldots, \frac{1}{2}}_{2 g \text { times }}, \underbrace{-1, \ldots,-1}_{r \text { times }}, \underbrace{1, \ldots, 1}_{s \text { times }}, \underbrace{1+r-g-s}_{=2-\sum_{i} q_{i}}, 1 ; \\
\left.\frac{1}{x_{1}}, \ldots, \frac{1}{x_{2 g-1}}, \frac{1}{p_{1}}, \ldots, \frac{1}{p_{s}}, \frac{1}{\varpi}\right),
\end{gathered}
$$

with $n=2 g+r+s-1, Q=1+r-g-s$ and $\varpi$ the image of the zero mode absorbing multi pole at infinity. In the cases relevant to this paper, the given data for an arbitrary Riemann surface are its branch points as well as the poles and zeroes of its metric $\lambda_{\mathrm{SW}}$. Therefore, taking into account charge balance and absorption of zero modes, we find

$$
\begin{aligned}
\pi^{-1}\left\langle\left\langle\prod_{i=1}^{2 g+2} \mu\left(e_{i}\right) \prod_{j=1}^{r} J\left(z_{r}\right) \prod_{k=1}^{s} P\left(p_{k}\right): P^{Q}(\infty):\right\rangle\right\rangle \\
=\prod_{i=1}^{2 g+2}\left(\partial_{e_{i}} M\left(e_{i}\right)\right)^{\frac{1}{4}} \prod_{j=1}^{r}\left(\partial_{z_{j}} M\left(z_{j}\right)\right)^{-\frac{1}{2}} \prod_{k=1}^{s}\left(\partial_{p_{k}} M\left(p_{k}\right)\right)^{\frac{1}{2}}\left(\infty^{2} \partial_{\infty} M(\infty)\right)^{\frac{1}{2} Q} \\
\times \quad \prod_{i=4}^{2 g+2}\left(M\left(e_{i}\right)\right)^{-\frac{1}{2}} \prod_{j=1}^{r}\left(M\left(z_{j}\right)\right)^{1} \prod_{k=1}^{s}\left(M\left(p_{k}\right)\right)^{-1}(M(\infty))^{-Q} \\
\times \quad F_{D}^{(n+1)}(\underbrace{\frac{1}{2}, \ldots, \frac{1}{2}}_{2 g \text { times }}, \underbrace{-1, \ldots,-1}_{r \text { times }}, \underbrace{1, \ldots, 1}_{s \text { times }}, \underbrace{1+r-g-s}_{=2-\sum_{i} q_{i}}, 1 ; \\
\left.\quad \frac{1}{M\left(e_{4}\right)}, \ldots, \frac{1}{M\left(e_{2 g+2}\right)}, \frac{1}{M\left(z_{1}\right)}, \ldots, \frac{1}{M\left(z_{r}\right)}, \frac{1}{M\left(p_{1}\right)}, \ldots, \frac{1}{M\left(p_{s}\right)}, \frac{1}{M(\infty)}\right),
\end{aligned}
$$

with again $n=2 g+r+s-1$ and $(1) r+(-1) s+\left(-\frac{1}{2}\right)(2 g+2)+2$ the total number of zero modes. Note the appearance of an additional crossing ratio which accounts for the zero mode absorbing terms at infinity.

A particular useful identity is the following expansion of $F_{D}$ in terms of hypergeometric functions, which can be used to find the analytic continuations exhibiting logarithmic divergencies:

$$
\begin{aligned}
& F_{D}^{(n)}\left(a, b_{1}, \ldots, b_{n}, c ; x_{1}, \ldots, x_{n}\right) \\
& =\sum_{m_{1}=0}^{\infty} \ldots \sum_{m_{k-1}=0}^{\infty} \sum_{m_{k+1}=0}^{\infty} \ldots \sum_{m_{n}=0}^{\infty} \frac{(a)_{m_{1}+\ldots+m_{k-1}+m_{k+1}+\ldots+m_{n}} \prod_{l \neq k}\left(b_{l}\right)_{m_{l}}}{(c)_{m_{1}+\ldots+m_{k-1}+m_{k+1}+\ldots+m_{n}} \prod_{l \neq k}(1)_{m_{l}}} \\
& \quad \times \prod_{l \neq k} x_{l}^{m_{l}}{ }_{2} F_{1}\left(a+\sum_{l \neq k} m_{l}, b_{k} ; c+\sum_{l \neq k} m_{l} ; x_{k}\right),
\end{aligned}
$$

valid for $1 \leq k \leq n$. Further information on the Lauricella function $F_{D}$, in particular on its analytical continuation properties, can be found, for example, in [19].

\section{References}

[1] N. Akerblom and M. Flohr, in preparation.

[2] Ph.C. Argyres and A.E. Faraggi, Phys. Rev. Lett. 74, 3931 (1995) hep-th/9411057.

[3] A.A. Belavin, A.M. Polyakov and A.B. Zamolodchikov, Nucl. Phys. B 241, 333 (1984).

[4] D. Berenstein, R. Corrado, W. Fischler, S. Paban and M. Rozali, Phys. Lett. B 384, 93 (1996) hep-th/9605168.

[5] D. Bernard, Z. Maassarani and P. Mathieu, Mod. Phys. Lett. A 12, 535 (1997) hep-th/9612217.

[6] M. Bershadsky and A. Radul, Int. J. Mod. Phys. A 2, 165 (1987). 
[7] A. Bilal and I.I. Kogan, Gravitationally dressed conformal field theory and emergence of logarithmic operators, hep-th/9407151, Nucl. Phys. B 449, 569 (1995) hep-th/9503209.

[8] R. Blumenhagen, W. Eholzer, A. Honecker, K. Hornfeck and R. Hübel, Phys. Lett. B 332, 51 (1994) hep-th/9404113; Int. J. Mod. Phys. A 10, 2367 (1995) hep-th/9406203.

[9] A. Brandhuber and S. Stieberger, Nucl. Phys. B 488, 199 (1997) hep-th/9610053.

[10] A. Cappelli, P. Valtancoli and L. Vergnano, Nucl. Phys. B 524, 469 (1998) hep-th/9710248.

[11] J.-S. Caux, I.I. Kogan, A. Lewis and A.M. Tsvelik, Nucl. Phys. B 489, 469 (1997) hep-th/9606138.

[12] J.-S. Caux, I.I. Kogan and A.M. Tsvelik, Nucl. Phys. B 466, 444 (1996) hep-th/9511134.

[13] J.S. Caux, N. Taniguchi and A.M. Tsvelik, Nucl. Phys. B 525, 671 (1998) 671 cond-mat/9801055.

[14] L.J. Dixon, D. Friedan, E.J. Martinec and S.H. Shenker, Nucl. Phys. B 282, 13 (1987).

[15] B. Duplantier and H. Saleur, Nucl. Phys. B 290, 291 (1987).

[16] W. Ehozler and M.R. Gaberdiel, Commun. Math. Phys. 186, 61 (1997) hep-th/9601163.

[17] J. Ellis, N.E. Mavromatos and D.V. Nanopoulos, Int. J. Mod. Phys. A 12, 2639 (1997) hep-th/9605046.

[18] H. Exton, Multiple Hypergeometric Functions and Applications, (Chichester: Ellis Horwood etc., 1976).

[19] H. Exton, q-Hypergeoemtric Functions and Applications, (Chichester: Ellis Horwood etc., 1983); Handbook of Hypergeometric Integrals: Theory, Applications, Tables, Computer Programs, (Chichester: Ellis Horwood etc., 1978).

[20] B.L. Feigin and D.B. Fuks, Funct. Anal. Appl. 17, 241 (1983); Verma Modules over the Virasoro Algebra, in Topology, Proc. Leningrad 1982, L.D. Faddeev and A.A. Mal'cev (eds.), Lect. Notes Math. 1060, 230 (1984), Springer Verlag.

[21] G. Felder, Nucl. Phys. B 317, 215 (1989) [Erratum-ibid. B 324, 548 (1989)].

[22] G. Felder, J. Fröhlich and G. Keller, Commun. Math. Phys. 124, 647 (1989).

[23] J. Fjelstad, J. Fuchs, S. Hwang, A.M. Semikhatov and I.Y. Tipunin, Nucl. Phys. B 633, 379 (2002) hep-th/0201091.

[24] M.A.I. Flohr, Commun. Math. Phys. 157, 179 (1993) hep-th/9207019; Mod. Phys. Lett. A 9, 1071 (1994) hep-th/9312097.

[25] M.A.I. Flohr, Int. J. Mod. Phys. A 11, 4147 (1996) hep-th/9509166; Int. J. Mod. Phys. A 12, 1943 (1997) hep-th/9605151.

[26] M.A.I. Flohr, Mod. Phys. Lett. A 11, 55 (1996) hep-th/9605152.

[27] M.A.I. Flohr, Nucl. Phys. B 482, 567 (1996) hep-th/9606130.

[28] M.A.I. Flohr, Nucl. Phys. B 514, 523 (1998) hep-th/9707090; Null vectors in logarithmic conformal field theory, hep-th/0009137.

[29] M.A.I. Flohr, Phys. Lett. B 444, 179 (1998) hep-th/9808169.

[30] M.A.I. Flohr, Nucl. Phys. B 634, 511 (2002) hep-th/0107242.

[31] M.A.I. Flohr, Int. J. Mod. Phys. A 18, 4497 (2003) hep-th/0111228.

[32] M.A.I. Flohr, The Rational Conformal Quantum Field Theories in Two Dimensions with Effective Central Charge $c_{\text {eff }} \leq 1$, preprint BONN-IR-94-11, (Ph.D. thesis in German, 1994).

[33] M.R. Gaberdiel and H.G. Kausch, Nucl. Phys. B 477, 293 (1996) hep-th/9604026; Phys. Lett. B 386, 131 (1996) hep-th/9606050.

[34] M.R. Gaberdiel, Nucl. Phys. B 618, 407 (2001) hep-th/0105046. 
[35] M.R. Gaberdiel, Int. J. Mod. Phys. A 18, 4593 (2003) hep-th/0111260.

[36] A.M. Ghezelbash and V. Karimipour, Phys. Lett. B 402, 282 (1997) hep-th/9704082.

[37] V. Gurarie, Nucl. Phys. B 410, 535 (1993) hep-th/9303160.

[38] V. Gurarie, M. Flohr and C. Nayak, Nucl. Phys. B 498, 513 (1997) cond-mat/9701212.

[39] V. Gurarie and A.W.W. Ludwig, J. Phys. A 35, L377 (2002) cond-mat/9911392.

[40] A. Hanany and Y. Oz, Nucl. Phys. B 452, 283 (1995) hep-th/9505075.

[41] E.V. Ivashkevich, J. Phys. A 32, 1691 (1999) cond-mat/9801183.

[42] H.G. Kausch, Phys. Lett. B 259, 448 (1991).

[43] H.G. Kausch, Curiosities at c=-2, hep-th/9510149

[44] H.G. Kausch, Nucl. Phys. B 583, 513 (2000) hep-th/0003029.

[45] A. Klemm, W. Lerche, S. Yankielowicz and S. Theisen, Phys. Lett. B 344, 169 (1995) hep-th/9411048.

[46] A. Klemm, W. Lerche and S. Theisen, Int. J. Mod. Phys. A 11, 1929 (1996) hep-th/9505150.

[47] A. Klemm, W. Lerche, P. Mayr, C. Vafa and N.P. Warner, Nucl. Phys. B 477, 746 (1996) 746 hep-th/9604034.

[48] V.G. Knizhnik, Phys. Lett. B 180, 247 (1986); Commun. Math. Phys. 112, 567 (1987).

[49] I.I. Kogan and A. Lewis, Nucl. Phys. B 509, 687 (1998) hep-th/9705240.

[50] I.I. Kogan, A. Lewis and O.A. Soloviev, Int. J. Mod. Phys. A 13, 1345 (1998) hep-th/9703028.

[51] I.I. Kogan, and N.E. Mavromatos, Phys. Lett. B 375, 111 (1996) hep-th/9512210.

[52] I.I. Kogan, N.E. Mavromatos and J.F. Wheater, Phys. Lett. B 387, 483 (1996) hep-th/9606102.

[53] I.I. Kogan, C. Mudry and A.M. Tsvelik, Phys. Rev. Lett. 77, 707 (1996) cond-mat/9602163.

[54] I.I. Kogan and A. Nichols, Int. J. Mod. Phys. A 17, 2615 (2002) hep-th/0107160.

[55] M. Krohn and M. Flohr, JHEP 0301, 020 (2003) hep-th/0212016; Fortsch. Phys. 52, 503 (2004) hep-th/0312185.

[56] W. Lerche, Nucl. Phys. Proc. Suppl. 55B, 83 (1997) [Fortsch. Phys. 45, 293 (1997)] hep-th/9611190.

[57] Z. Maassarani and D. Serban, Nucl. Phys. B 489, 603 (1997) hep-th/9605062.

[58] S. Moghimi-Araghi, S. Rouhani and M. Saadat, Int. J. Mod. Phys. A 18, 4747 (2003) hep-th/0201099.

[59] S. Nakamura, Recoiling D-branes, hep-th/0406193

[60] V. Periwal and O. Tafjord, Phys. Rev. D 54, 3690 (1996) hep-th/9603156.

[61] M.R. Rahimi Tabar, A. Aghamohammadi and M. Khorrami, Nucl. Phys. B 497, 555 (1997) hep-th/9610168.

[62] M.R. Rahimi Tabar and S. Rouhani, Annals Phys. 246, 446 (1996) hep-th/9503005; Nuovo Cim. B 112, 1079 (1997) hep-th/9507166; Europhys. Lett. 37, 447 (1997) hep-th/9606143; Logarithmic Correlation Functions in Two Dimensional Turbulence, hep-th/9606154

[63] M.R. Rahimi Tabar and S. Rouhani, Phys. Lett. B 431, 85 (1998) hep-th/9707060.

[64] F. Rohsiepe, On reducible but indecomposable representations of the Virasoro algebra, hep-th/9611160

[65] L. Rozansky and H. Saleur, Nucl. Phys. B 376, 461 (1992); Nucl. Phys. B 389, 365 (1993) hep-th/9203069.

[66] H. Saleur, Nucl. Phys. B 382, 486 (1992) hep-th/9111007; Nucl. Phys. B 382, 532 (1992) hep-th/9111008.

[67] J. Schulze and N.P. Warner, Nucl. Phys. B 498, 101 (1997) hep-th/9702012. 
[68] N. Seiberg, Phys. Lett. B 388, 753 (1996) hep-th/9608111.

[69] N. Seiberg and E. Witten, Nucl. Phys. B 431, 484 (1994) hep-th/9408099;

Nucl. Phys. B 426, 19 (1994) [Erratum-ibid. B 430 (1994) 485] hep-th/9407087.

[70] N. Seiberg and E. Witten, Gauge dynamics and compactification to three dimensions, hep-th/9607163

[71] A. Shafiekhani and M.R. Rahimi Tabar, Int. J. Mod. Phys. A 12, 3723 (1997) hep-th/9604007.

[72] S. Skoulakis and S. Thomas, Phys. Lett. B 438, 301 (1998).

[73] G.M.T. Watts, J. Phys. A 29, L363 (1996) cond-mat/9603167.

[74] Xiao-Gang Wen, Yong-Shi Wu and Yasuhiro Hatsugai, Nucl. Phys. B 422[FS], 476 (1994) cond-mat/9311038; Xiao-Gang Wen and Yong-Shi Wu, Chiral operator product algebra hidden in certain fractional quantum Hall wave functions, cond-mat/9310027.

[75] A.B. Zamolodchikov, Nucl. Phys. B 285, 481 (1987).

[76] A.B. Zamolodchikov, Nucl. Phys. B 358, 497 (1991). 